\title{
Investigaciones hidrogeológicas en el Parque Nacional de Ordesa y Monte Perdido (Huesca, España)
}

\author{
Luis Javier Lambán ${ }^{(1)}$, Jorge Jódar(1) y Emilio Custodio ${ }^{(2)}$
}

(1) Instituto Geológico y Minero de España, Unidad de Zaragoza, C/ Manuel Lasala, 44, 50006 Zaragoza, España. javier.lamban@igme.es, j.jodar@igme.es

(2) Grupo de Hidrología Subterránea, Departamento de Ingeniería Civil y Ambiental, Universidad Politécnica de Cataluña. C/ Jordi Girona, 1 - 3 UPC Campus Nord, Edificio D2, 08034 Barcelona, España. emilio.custodio@upc.edu

\section{RESUMEN}

El Parque Nacional de Ordesa y Monte Perdido (PNOMP) constituye el mayor macizo montañoso calcáreo de Europa Occidental. En él se encuentra el karst de mayor altitud de toda Europa. El agua subterránea resulta esencial en la génesis, desarrollo y evolución del Parque. Se obtiene una distribución espacial de la capacidad de infiltración aplicando el método APLIS y una estimación preliminar de la recarga promedio entre el 72 y $77 \%$ de la precipitación media anual mediante la aplicación de los códigos HBV y Visual Balan. Esto pone de manifiesto una elevada vulnerabilidad de los recursos de agua del PNOMP ante el posible cambio climático y global. El agua es mayoritariamente bicarbonatada cálcica y bicarbonatada cálcico magnésica. Se observa que predomina la disolución de calcita, en coherencia con los materiales carbonatados más importantes del Parque: Cretácico superior y Paleoceno Eoceno inferior. Otros procesos hidrogeoquímicos observados son la disolución de anhidrita y/o yeso, así como una posible disolución incongruente de dolomita en el Valle de Ordesa. Los isótopos estables del agua indican que los frentes que dejan precipitaciones más cuantiosas proceden del Océano Atlántico. En otoño, invierno y primavera se registra un exceso de deuterio en el agua de recarga que puede estar relacionado con la sublimación diurna de la nieve y la posterior condensación nocturna del vapor de agua atmosférico sobre el manto nival. La zona de recarga está comprendida entre 1950 y 2600 m snm y el agua de los principales manantiales del acuífero presenta un tiempo de transito relativamente corto, entre 1.1 y 4.5 años.

Palabras clave: Acuífero kárstico, Hidrogeoquímica e isótopos ambientales, Parque Nacional de Ordesa y Monte Perdido, Recarga, Tiempo de tránsito.

\section{Hydrogeological research in the Ordesa and Monte Perdido National Park (Huesca, Spain)}

\begin{abstract}
The National Park of Ordesa and Monte Perdido (PNOMP) is the largest mountainous limestone massif of Western Europe. It has the highest altitude karst of the whole Europe. Groundwater is essential for the genesis, development and evolution of the park. The spatial distribution of recharge infiltration capacity is obtained by using the APLIS method. Additionally, a preliminary estimation of recharge is obtained by using the HBV and Visual Balan codes. The values of average surface runoff, evapotranspiration and recharge for the whole Paleocene-Eocene aquifer in the park are respectively about 160, 360 and $1227 \mathrm{~mm} / \mathrm{yr}$, or 10, 20 and $70 \%$ of yearly precipitation. Average recharge ranges between 72 and $77 \%$ of yearly average precipitation. This highlights the high vulnerability of water resources of PNOMP to possible climate and global change, according to surface and deep karstification and the snow cover during several months a year. Water is mainly of the calcium bicarbonate and calcium-magnesium bicarbonate types. There is a dominant process of calcite dissolution, which is consistent with the carbonate nature of the most abundant materials in the park (Upper Cretaceous and Lower Paleocene Eocene). Other observed hydrogeochemical processes are anhydrite and/or gypsum dissolution, as well as a possible incongruent dissolution of dolomite in the Ordesa Valley. Water stable isotopes show that the fronts that produce the greatest precipitations come from the Atlantic Ocean. In autumn, winter and spring there is a deuterium excess in the recharge water which is assumed to be related with diurnal snow sublimation and night water vapour condensation over the snow pack. The recharge ele-
\end{abstract}


Luis Javier Lambán, et al., 2019. Investigaciones hidrogeológicas en el Parque Nacional... Boletín Geológico y Minero, 130 (4): 615-640

vation zone is located between 1950 and $2600 \mathrm{~m}$ asl, and most of the sampled springs show short transit times, between 1 and 4 years, although one of the springs (Fuen dero Baño) shows the influence of a slowly changing regional groundwater flow system.

Keywords: aquifer, karst, Pyrenees, recharge, hydrogeochemical, environmental isotopes, transit time, Ordesa and Monte Perdido National Park.

\section{ABRIDGED ENGLISH VERSION}

\section{Introduction}

The Ordesa and Monte Perdido National Park (PNOMP), located in the Pyrenees, is the highest calcareous massif in Western Europe. Its landscape responds mainly to glacial and karst processes that allow us to consider the PNOMP as a natural laboratory for analysing the dynamics of high altitude karst hydrogeological systems. Despite its importance, the hydrogeological characterization of the PNOMP is still limited (CHE, 1998, 2001; Ríos-Aragüés, 2003; Lambán et al., 2015)

Karst aquifers have characteristics that make them very different from other aquifers, such as their high heterogeneity generated by the endokarstic network, the high velocity of the water flow and short residence times (e.g. Kiraly, 1997; Motyka, 1998). When karst aquifers are located in high altitude mountain zones, their hydrogeologic response is influenced by other variables, including important vertical temperature gradients, abrupt topography that produces extremely short response times to precipitation events, and snow melting dynamics that control aquifer recharge during the winter and spring seasons (Gremaud et al., 2009; Gremaud and Goldscheider 2010). In these cases, the analysis of environmental isotopes represents a powerful tool for characterizing their hydrogeology (Collins and Gordon, 1981). Thus, recharge zones can be defined by using $\delta^{18} \mathrm{O}$ and $\delta^{2} \mathrm{H}$ (Araguás-Araguás et al., 2000; Gonfiantini et al., 2001), tritium can be either used to separate base flow from runoff resulting from snow melt or to estimate groundwater transit times (Boronina et al., 2005; Gustafson, 2007), and $\delta^{34} S_{S_{04}}$ and $\delta^{18} \mathrm{O}_{504}$ can be used to detect different groundwater origins (Schaefer and Usdowski, 1992; González-Fernández et al., 2009) in the discharge of the springs belonging to the karst system.

Despite the significance of high altitude karst hydrogeological systems which are present in the main European main alpine ranges (i.e. the Alps, Pyrenees and Carpathians), the hydrogeological behaviour of such systems is indeed scarce. The objective of this study is to present a summary of the hydrogeological investigations conducted by the Geological and Mining Institute of Spain (IGME) in collaboration with the Confederación Hidrográfica del Ebro (CHE) in the PNOMP. It includes (1) a preliminary evaluation of the spatially distributed infiltration capacity, (2) the estimation of the aquifer system recharge obtained by conducting a soil-water balance (HBV and Visual-Balan numerical models), (3) the hydrochemical and environmental isotopic tracer characterization of both, groundwater and rainfall in the PNOMP.

\section{Study zone}

The PNOMP is located in the central sector of the Pyrenees, the most important mountain chain of the Iberian Peninsula (Fig. 1). The maximum altitude corresponds to the Monte Perdido, reaching $3355 \mathrm{~m}$ asl (above sea level). It is one of the highest peaks of the entire Pyrenean range.

From a geological point of view (Fig. 2), the most important tectonic characteristic is the presence of two big units: Gavarnie and Monte Perdido. The first one is observed at the northern part of the study area and includes the Sierra Tendeñera (Paleozoic and upper Cretaceous outcroppings). The second one includes Cretaceous to lower Eocene outcroppings. It is characterised by a strong plunging of these materials towards the south, under the Eocene flysch. This unit presents scarce internal deformation, which prevents the local hydraulic connection between the different permeable levels in the lower part of the Ordesa Valley. The limestones, dolomites and calcarenites of the Upper Cretaceous and the Paleocene-Eocene constitute the most important surficial permeable levels and sustain the most significant springs and water discharges. The main karst system is located on the lower Paleocene-Eocene materials.

From a climatic point of view, the PNOMP has a cold climate with a dry season, with mild and cool summers and significant altitudinal variations. At the Fanlo-Góriz meteorological station (P1, Table 1), located at $2,200 \mathrm{~m}$ asl, the mean annual temperature is $4.9^{\circ} \mathrm{C}$ and the average precipitation is $1650 \mathrm{~mm} / \mathrm{yr}$. Rainfall originating from the Atlantic Ocean is the main source of aquifer recharge in the PNOMP (Lambán et al., 2014). 
Luis Javier Lambán, et al., 2019. Investigaciones hidrogeológicas en el Parque Nacional... Boletín Geológico y Minero, 130 (4): $615-640$

\section{Materials and methods}

Measured data

To characterize the hydrological functioning of the PNOMP, water samples from rainfall and groundwater are periodically collected (Table 1). Figure 1 shows the geographic location of the sampled meteorological stations (Fig. 3) and springs.

The discharge of the Arazas River is measured at the outlet of the PNOMP in the gauging station located on La Ereta bridge (Fig. 4). In this point the river integrates the runoff of the whole Ordesa Valley. Very close to this point the piezometer of Bujaruelo is located, which is the only point where the piezometric evolution of the aquifer is measured.

\section{Rainfall infiltration capacity}

To evaluate the spatial distribution of the rainfall infiltration capacity $(\mathrm{Cl})$ in the PNOMP, a modification of the APLIS method has been applied. The APLIS method (Andreo et al., 2008) is typically used for assessing the infiltration capacity in carbonated aquifers from the following intrinsic variables of the territory: altitude (A), slope (P), lithology (L), preferential infiltration zones (I) and soils (S). By means of Geographic Information Systems (GIS), each layer of information is classified according to the spatial distribution of these variables (i.e. $A, P, L, I, S)$ throughout the study zone. Each variable is evaluated in the study zone, assigning values of between 1 (negligible) and 10 (principal), depending on the role played by these variables in allowing infiltration. Then, these variable layers are combined to obtain the infiltration capacity map (Fig. 5) according to the following equation: $\mathrm{Cl}=(A+P+3 L+2 I+S) / 0.9$.

\section{Aquifer recharge evaluation}

The aquifer recharge has been numerically estimated by using two different modelling platforms: HBV (Seibert, 1997, 2005) and Visual Balan (Samper et al., 2005), and the groundwater level as state variable. In both cases the corresponding model parameters have been calibrated so the model reproduces the evolution of the piezometric head observed in the piezometer of Bujaruelo.

\section{Transit time $\tau$ and recharge altitude estimation}

The method used to estimate $\tau$ and $Z_{R}$ takes profit of the observed variation of both, the amplitude of the seasonal isotopic composition of precipitation $A_{i n}(Z)$ and the mean isotopic content in rainfall along a vertical transect $\delta_{i n}(Z)$. Once and $A_{i n}(Z)$ are characterized, it is possible to estimate the spring altitude recharge $Z_{R}$, then the corresponding amplitude of the seasonal isotopic content in rainfall at that altitude $A_{\text {in }}(Z)$, and finally the mean transit time $\tau$ as a function of the amplitude dampening $f$, which is defined as the ratio between the amplitude of the seasonal isotopic content in the sampled groundwater $A_{\text {out }}\left(Z_{\text {spring }}\right)$ and $A_{\text {in }}\left(Z_{R}\right)(J$ ódar et al., 2016b).

\section{Results}

The recharge infiltration capacity obtained with the APLIS method is high throughout the PNOMP, ranging from 40 to $60 \%$ of total precipitation. It shows a slight increase with elevation which is conditioned upon both the more intense karst development and the reduction of vegetation with elevation. This result is in line with what is obtained by means of the conceptual numerical models HBV and VisualBalan (Fig. 6), which yield groundwater recharge corresponding to $80 \%$ and $70 \%$ of precipitation, respectively. In both cases, the simulated piezometric evolution resembles the observed one (Figs. 7 and 8). Although the obtained recharge values are high, they make sense if the hydrogeological setting of the study area is taken into account. These results are coherent with those obtained in other similar areas, such as the Beceit-Tortosa Ports (Espinosa and Custodio, 2014). The estimated storage coefficient $S$ ranges between 0.17 and 0.33 for HBV and is 0.28 for Visual Balan. These values are larger than the expected for this type of aquifer. More research is needed to address this issue.

Groundwater in the PNOMP typically shows a calcium-bicarbonate or calcium-magnesium-bicarbonate composition (Fig. 9), which is coherent with the carbonate nature of the main permeable materials (upper 
Luis Javier Lambán, et al., 2019. Investigaciones hidrogeológicas en el Parque Nacional... Boletín Geológico y Minero, 130 (4): $615-640$

Cretaceous and lower Paleocene-Eocene limestones, dolomites and calcarenites) found in the study zone. Nevertheless, two additional hydrogeochemical facies can also be found: (1) calcium-sulphate composition in the Fuen I Abellá (site 10), Fuen Roya Aguas Abajo (site 5), and Fuen Roya-Surgencia (site 6) springs; these three springs are located in nearby sectors, suggesting their probable connection and a dominant anhydrite and/or gypsum dissolution process (Figs. 10 and 11), and (2) sodium-sulphate-chloride composition in the Fuen dero Baño spring (site 22), with an EC of $3600 \mu \mathrm{S} / \mathrm{cm}$ and $T$ of $26^{\circ} \mathrm{C}$, measured in situ, that clearly contrasts with the mean $E C$ of $400 \mu \mathrm{S} / \mathrm{cm}$ and a $T$ of $10.5^{\circ} \mathrm{C}$ measured in other springs of this area.

The isotopic $\left(\delta^{18} \mathrm{O}\right)$ content in rainfall ranges between -4.5 and $-15 \%$, whereas in the springs it varies between -7 and-12 \% (Fig. 12). In both cases the isotopic shows a clear seasonal pattern (Fig. 13). During autumn, winter and spring there is a deuterium excess, which may be related to snow sublimation and its later condensation on the snow surface. In the case of rainfall, the local altitudinal isotopic gradient is $2.2 \% / \mathrm{km} \delta^{18} \mathrm{O} / 100 \mathrm{~m}$, which is coherent with the gradient values obtained for different alpine mountainous zones. The amplitude of the seasonal isotopic content in rainfall $A_{\text {siooin }}$ and elevation show an increasingly monotonic relationship pointing towards the existence of a major common source of moisture for the three sampled meteorological stations in the PNOMP. This result is coherent with the findings of Lambán et al. (2015), who found that precipitation in the PNOMP comes mostly from the Atlantic Ocean. The vertical gradient of the seasonal isotopic amplitude $\nabla_{z} A_{s 180 i n}$ is $0.9 \% / \mathrm{km}$, which is close to that obtained from a vertical transect in the Alps (Jódar et al., 2016a).

The aquifer is assumed to behave as an exponential-piston flow system. The estimated transit times are short (Fig. 15A), ranging between $1.12 \mathrm{yr}$ (site 18) and $4.48 \mathrm{yr}$ (site 6A), with an average value of $1.85 \mathrm{yr}$ and a standard deviation of $0.8 \mathrm{yr}$. The springs with the shortest transit time (sites 18, 19, 23 and 9) correspond to those springs discharging groundwater from the more karstified materials of Eocene-Paleocene age. The springs with the longest transit time (sites $6 A, 6 B, 4,42,43$ and 44) correspond to springs discharging from the Upper Cretaceous materials.

The volume associated to the exponential flow transit time distribution increases as the hydrologic system transit time increases, and conversely (Fig. 15B). As the transit time increases, the length of the aquifer flow lines increases accordingly. This favours the transition towards a perfect mixing distribution of transit times, with the role played by the piston flow transit time distribution in the hydrological system response vanishing.

\section{Conclusions}

Groundwater in the PNOMP is mainly composed of the calcium bicarbonate and calcium-magnesium bicarbonate types, suggesting a dominant calcite dissolution process in agreement with the dominant lithology. Additionally, two other minor chemical compositions can be found: a sodium-sulphate-chloride and a calcium-sulphate composition.

Rainfall infiltration capacity of the different materials composing the PNOMP is high and consequently recharge form precipitation is high as well. It has been numerically estimated to be between $70 \%$ and $80 \%$ of total precipitation.

The local altitudinal isotopic gradient is $-2.2 \% / \mathrm{km} \delta^{18} \mathrm{O} / 100 \mathrm{~m}$. There is a linear relationship between the amplitude of the seasonal isotopic content of rainfall and elevation, with a vertical gradient of $0.9 \% / \mathrm{km}$ that confirms the Atlantic Ocean as the major source of moisture for precipitation in the PNOMP. The apparent recharge zone for the sampled springs is located at an elevation ranging between 2,600 and 1,950 $\mathrm{m}$ asl. The hydrogeological system presents short transit times, in agreement with the karstic nature of the aquifer system. The obtained transit time distribution points to increased mixing the longer the system transit time is. This extends the recharge zone to a broader zone along the slope.

\section{Introducción}

Los acuíferos kársticos tienen características particulares que los hacen muy diferentes de otros acuíferos. Tales son la alta heterogeneidad generada por la propia red endokárstica, las elevadas velocidades de tránsito del agua y los pequeños tiempos de residencia del agua en el sistema (Kiraly, 1997; Motyka, 1998). En zonas de alta montaña, la dinámica de estos acuí- feros está afectada por los efectos asociados a los relieves abruptos y grandes altitudes, tiempos de respuesta a episodios de precipitación extremadamente cortos e importantes gradientes verticales de temperatura. Además, la fusión de la nieve controla la recarga durante el invierno y la primavera (Gremaud et al., 2009; Gremaud y Goldscheider, 2010). Acompañando a las técnicas de observación y exploración hidrogeológica tradicionales, las técnicas hidrogeoquímicas y 
en particular las isotópicas ambientales son muy útiles para caracterizar la dinámica y el desarrollo del modelo conceptual de este tipo de acuíferos (Collins y Gordon, 1981). Las zonas de recarga pueden definirse mediante el $\delta^{18} \mathrm{O}$ y $\delta^{2} \mathrm{H}$ del agua (AraguásAraguás et al., 2000; Gonfiantini et al., 2001). El contenido en tritio $\left({ }^{3} \mathrm{H}\right)$ del agua puede utilizarse para separar el caudal de base del producido por la fusión de la nieve, así como para estimar el tiempo de tránsito del agua subterránea (Boronina et al., 2005; Gustafson, 2007). Por otro lado, el $\delta^{34} S\left(\mathrm{SO}_{4}\right)$ y $\delta^{18} \mathrm{O}$ $\left(\mathrm{SO}_{4}\right)$ pueden aportar información sobre el origen de los solutos y de los procesos de interacción del agua con la roca (Schaefer y Usdowski, 1992; GonzálezFernández et al., 2009).

A pesar de la importancia de los acuíferos kársticos situados en zonas de alta montaña (como por ejemplo en los Alpes, los Pirineos y/o los Cárpatos), la caracterización hidrogeológica de estos sistemas complejos no se ha estudiado extensivamente. En el presente documento se presenta un resumen de las investigaciones hidrogeológicas que viene realizando el Instituto Geológico y Minero de España (IGME) en el PNOMP desde 2007, en parte en colaboración con la Confederación Hidrográfica del Ebro (CHE). Se incluyen las primeras estimaciones de la capacidad de infiltración obtenidas mediante la aplicación de una modificación del método APLIS y se presenta una evaluación preliminar de la recarga del acuífero obtenida mediante un balance del agua en el suelo (códigos HBV y Visual Balan). Se incorpora así mismo la caracterización hidrogeoquímica e isotópica tanto de la precipitación (Iluvia y nieve) como de las aguas subterráneas, estableciendo los procesos hidrogeoquímicos dominantes que resultan de la interacción agua-roca y se estima tanto la altitud de recarga como el tiempo medio de tránsito mediante la aplicación de técnicas isotópicas ambientales. Por último, se resumen otras investigaciones relacionadas con las aguas subterráneas en el PNOMP.

\section{Marco geográfico y climático}

EI PNOMP se ubica al noreste de la Península lbérica, en el sector central de la Cordillera Pirenaica y más concretamente dentro de la comarca del Sobrarbe, de Huesca, en Aragón (Figura 1). La zona de protección del parque limita al norte con los valles de Gavarnie y Estaubé, en Francia, al sur con el barranco San Chaime, al este con el valle de Pineta y la garganta de Escuaín y al oeste con el valle de Bujaruelo. Tiene una superficie de 15.608 hectáreas (Benito Alonso, 2006). La zona periférica del PNOMP es bastante más amplia y la conforman el valle del Bujaruelo (cabecera del río Ara), la solana del valle Vió, la margen derecha del barranco de Airés en el valle de Puértolas, la parte media del valle de Pineta y toda la cuenca del río La Larri hasta los lagos y picos de La Munia (Benito Alonso, 2006).

El PNOMP está compuesto por un sistema de valles y cumbres enlazados, destacando las gargantas radiales que dibujan los principales cursos fluviales: Cañón de Añisclo al S, desde Punta de las Olas a la fuente termal del Baño, Valle de Escuaín al SE, desde la Gran Suca o Pico de Añisclo a Revi-lla, Valle de Ordesa al O, desde la cumbre de Monte Perdido hasta el Puente de los Navarros, y Valle de Pineta al OSE, desde la cumbre de Monte Perdido hasta las Inglatas. La morfología de los límites del PNOMP en planta parece arbórea, donde el río Bellós dibuja un tronco diferenciado y una copa compuesta por los valles radiales más cortos del río Yaga, Arazas y Cinca (Martínez de Pisón, 2002). La altitud máxima en el PNOMP corresponde al Monte Perdido, a $3355 \mathrm{~m} \mathrm{snm}$ (sobre el nivel del mar), que es el tercer pico más alto de toda la cordillera Pirenaica. El punto situado a menor altitud del PNOMP coincide con el manantial de Fuen dero Baño (punto 22, Figura 1, Tabla 1), situado a $689 \mathrm{~m}$ snm. EI PNOMP lo conforman los municipios oscenses de Torla, Fanlo, Puértolas, Tella-Sin y Bielsa, de los cuales ninguno tiene su núcleo de población en el interior del Parque. Son municipios con un bajo número de habitantes empadronados, entre los 118 de Fanlo y los 496 de Bielsa, aunque la población se incrementa notablemente en temporadas vacacionales.

Desde un punto de vista climático y según la clasificación de Köppen-Geiger (Peel et al., 2007), el PNOMP tiene un clima frío, con una estación seca, con veranos suaves y frescos. Presenta variaciones térmicas altitudinales significativas. En la estación meteorológica de Fanlo-Góriz (punto P1, Tabla 1), que se encuentra a $2200 \mathrm{~m} \mathrm{snm}$, la temperatura media anual es de $4.9^{\circ} \mathrm{C}$ y la precipitación media de $1650 \mathrm{~mm} /$ año. Los meses de julio y agosto son los más cálidos y los de enero y febrero los más fríos, siendo en estos últimos meses en los que se concentran las mayores heladas. Según los datos meteorológicos disponibles, el promedio de nevadas en Góriz es de 52 días al año, pero, al igual que ocurre con las lluvias, hay una gran variabilidad interanual. En general, las nevadas comienzan en noviembre y suelen durar hasta mediados de mayo (Polo, 2015). Las medias mensuales de la precipitación presentan dos máximos durante el otoño ( $220 \mathrm{~mm}$ en octubre) y la primavera ( $185 \mathrm{~mm}$ en mayo) y tiene dos mínimos durante el invierno (80 mm en febrero) y el verano (105 mm en julio) (Lambán 
et al., 2015) (Figura 2). Las variabilidad espacial de la precipitación presenta un gradiente O-E (Benito Alonso, 2006) debido a los frentes atmosféricos de baja presión que llegan desde el Atlántico. Ellos son los responsables de los principales volúmenes de precipitación registrados en el PNOMP (Lambán et al., 2015). En la zona de estudio, los gradientes verticales promedio de temperatura y precipitación son de -3.3 ${ }^{\circ} \mathrm{C} / \mathrm{km}$ y $200 \mathrm{~mm} / \mathrm{km}$, respectivamente.

La ubicación de los numerosos lugares citados que no están en la Figura 1 pueden encontrarse en la Guía de Visita del Parque Nacional de Ordesa y Monte Perdido (Rada-Martínez et al., 2011) o en la cartografía del Instituto Geográfico Nacional (Hojas 146 y 178 a escala 1:25000)

\section{Marco geológico e hidrogeológico}

Desde un punto de vista geológico, el macizo pirenaico es un cinturón alpino resultante de la colisión entre las placas Ibérica y Europea (Muñoz, 1992).
Presenta una superficie aproximada de unos 1000 kilómetros de largo y entre 175 a 250 km de ancho. Las rocas que afloran en este macizo pueden agruparse en dos categorías: el basamento pre-hercínico, con edades desde el Precámbrico hasta el Carbonífero medio-superior, y la cobertera post-hercínica, con edades comprendidas desde el Estefaniense hasta el Terciario. Las rocas pre-hercínicas han sido afectadas por dos importantes acontecimientos orogénicos: el Hercínico (finales del Carbonífero, preEstefaniense) y las orogenias alpinas (EocenoOligoceno). La tectónica Hercínica se caracteriza por una foliación regional relacionada con pliegues de orientación ONO-ESE, zonas de fractura con orientaciones N-S y NE-SO y varias estructuras de cabalgamiento de orientación E-O (Carreras y Santanach, 1983). Este marco estructural fue heredado de cuando formaron los modernos Pirineos durante la orogenia Alpina (Parish, 1984; Muñoz et al., 1986). Desde finales del Cretácico hasta el Mioceno se ha desarrollado un orógeno asimétrico y doblemente vergente, caracterizado por un duplex central con un basamento her-
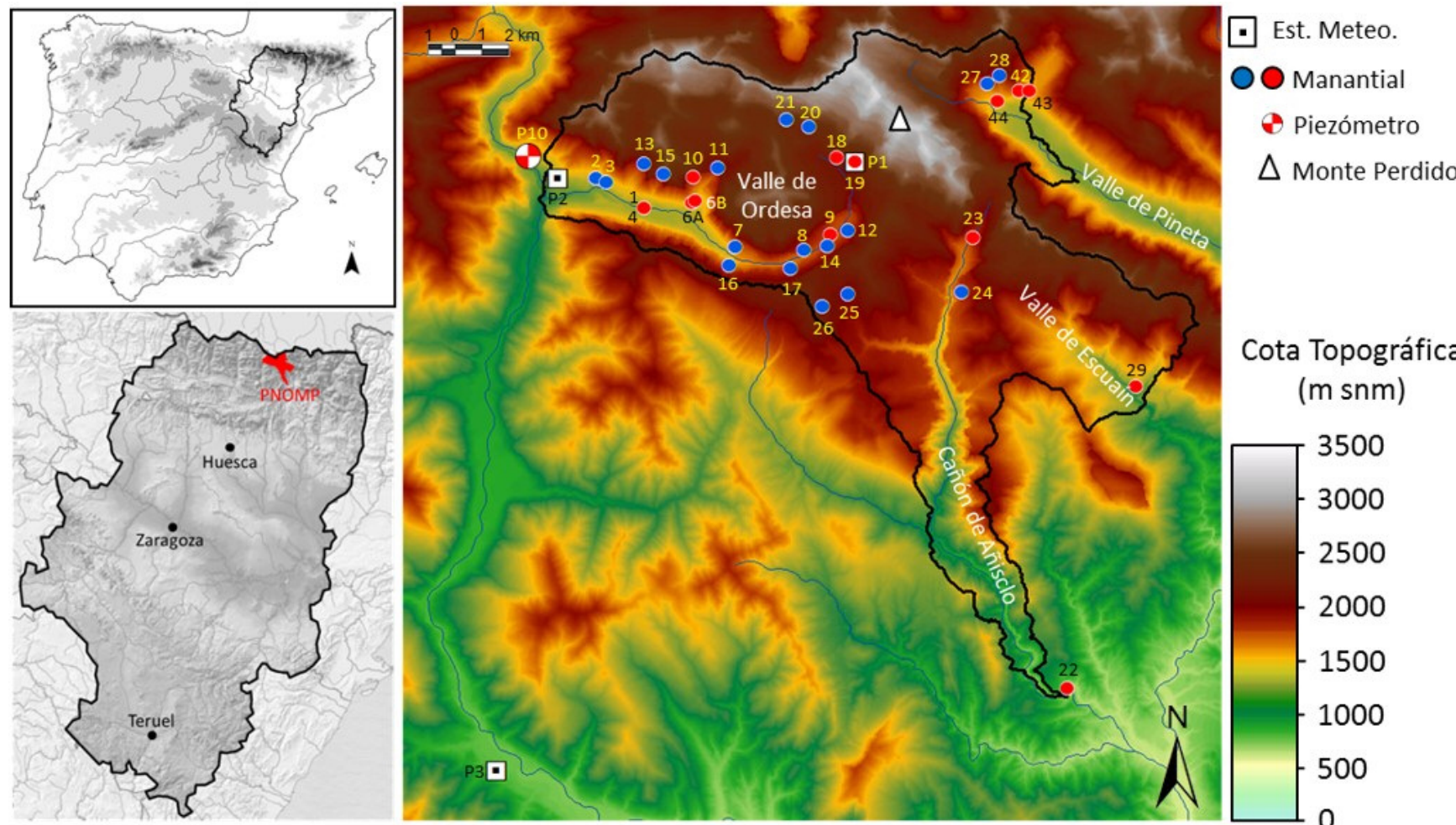

Figura 1. Situación del Parque Nacional de Ordesa y Monte Perdido. Los cuadrados corresponden a las estaciones meteorológicas y los ć́rculos a los manantiales de la zona de estudio. Los manantiales muestreados periódicamente incluidos en la red de monitoreo del PNOMP están marcados en rojo. Los códigos corresponden a los de la Tabla 1.

Figure 1. Location map of the Ordesa and Monte Perdido National Park. The squares correspond to meteorological stations and the circles to springs. The periodically sampled springs included in the monitoring network of the PNOMP are in red colour. The codes correspond to those in Table 1. 
Luis Javier Lambán, et al., 2019. Investigaciones hidrogeológicas en el Parque Nacional... Boletín Geológico y Minero, 130 (4): $615-640$

\begin{tabular}{|c|c|c|c|c|c|c|}
\hline Código punto & Tipo de punto & Nombre & Lon $\left({ }^{\circ}\right)$ & Lat $\left({ }^{\circ}\right)$ & Z (m snm) & Muestreo periódico \\
\hline $\mathrm{P} 2$ & Est. Meteo. & Parador & -0.097628 & 42.657177 & 1216 & Sí \\
\hline P3 & Est. Meteo. & Fiscal & -0.112950 & 42.488675 & 772 & Sí \\
\hline P10 & Piezómetro & Bujaruelo & -0.102831 & 42.658879 & 1080 & Sí \\
\hline 4 & Manantial & As Fuens & -0.064074 & 42.649752 & 1349 & Sí \\
\hline $6 \mathrm{~A}$ & Manantial & Fuen Roya 1 & -0.045506 & 42.651597 & 1348 & Sí \\
\hline $6 \mathrm{~B}$ & Manantial & Fuen Roya 2 & -0.044245 & 42.652465 & 1358 & Sí \\
\hline 10 & Manantial & Fuen I'Abellana & -0.045391 & 42.658933 & 1750 & Sí \\
\hline 11 & Manantial & Fuen Cotatuero & -0.036008 & 42.662022 & 1800 & No \\
\hline 12 & Manantial & Fuen De Soaso & 0.014654 & 42.645481 & 1820 & No \\
\hline 13 & Manantial & Fuen Carriata & -0.064759 & 42.662150 & 1820 & No \\
\hline 14 & Manantial & Fuen de Soaso & 0.007289 & 42.640965 & 1830 & No \\
\hline 15 & Manantial & Fuen Gallinero & -0.056822 & 42.659443 & 1840 & No \\
\hline 16 & Manantial & Fuen Freda & -0.030267 & 42.634247 & 1860 & No \\
\hline 17 & Manantial & Fuen de L'Abe & -0.006619 & 42.634018 & 1920 & No \\
\hline 23 & Manantial & Font Blanca & 0.063073 & 42.644637 & 1710 & Sí \\
\hline 24 & Manantial & Fuen dero Foratiello & 0.059288 & 42.629142 & 1790 & No \\
\hline 25 & Manantial & Fuen dero Furicon & 0.015649 & 42.627445 & 2010 & No \\
\hline 26 & Manantial & Fuen dero Esmoladera & 0.006009 & 42.623503 & 2050 & No \\
\hline 27 & Manantial & Fuen el Felcarral & 0.066618 & 42.688439 & 1703 & No \\
\hline 28 & Manantial & Fuen la Bispera & 0.071265 & 42.690844 & 1930 & No \\
\hline 29 & Manantial & Fuen de Escuaín & 0.127760 & 42.604122 & 1050 & Sí \\
\hline 42 & Manantial & Sucarraz & 0.078970 & 42.686720 & 1468 & Sí \\
\hline 43 & Manantial & Os Churros & 0.082853 & 42.686751 & 1488 & Sí \\
\hline 44 & Manantial & Esquinarasnos & 0.070821 & 42.683572 & 1449 & Sí \\
\hline
\end{tabular}

Tabla 1. Estaciones meteorológicas instaladas, piezómetro y manantiales muestreados existentes en el PMOMP (Lambán et al., 2015). Las estaciones meteorológicas y el piezómetro se muestrean mensualmente y los manantiales de la red de observación con una periodicidad aproximadamente trimestral.

Table 1. Installed meteorological stations, piezometer and springs sampled in the PNOMP (Lambán et al., 2015). The meteorological stations and also the piezometer are sampled on a monthly basis and the springs of the monitoring network are sampled approximately quarterly. 
cínico (zona axial), flanqueado por el norte y el sur por pliegues y cabalgamientos asociados a las cuencas de antepaís (Muñoz, 1992).

EI PNOMP forma parte del anteriormente mencionado sistema imbricado de pliegues y cabalgamientos vergentes al sur (Seguret, 1972). La arquitectura estructural del PNOMP responde a la existencia de dos mantos de corrimiento principales: el manto de Gavarnie, al O del Río Ara, y el manto del Monte Perdido, hacia el E (Seguret, 1972). El manto de Gavarnie se observa en la parte norte del área de estudio, donde afloran los materiales del Paleozoico y del Cretácico superior. El manto del Monte Perdido comprende materiales desde el Cretácico hasta el Eoceno inferior, presentando una estructura de estratificación sub-horizontal con escasa deformación interna en la parte inferior del Valle de Ordesa. Esta escasa deformación, unida a la intercalación de formaciones menos permeables, favorece localmente la desconexión hidráulica de los distintos niveles permeables (CHE, 1998). Hacia el sur, estos materiales presentan mayor inclinación y subyacen bajo turbiditas del Eoceno medio. (Figura 2).

EI PNOMP presenta un paisaje de montaña alpina, el cual es el resultado de un intenso proceso de karstificación y de la erosión debida a procesos glaciales. Los efectos de la karstificación se dejan notar por la presencia de numerosas zonas de lapiaz, cuevas, sumideros y fisuras. Por otro lado, los procesos glaciales se reflejan en los largos y profundos valles en forma de $U$ de Ordesa, Añisclo, Escuaín y Pineta (IGME-OAPN, 2013).

Desde un punto de vista hidrogeológico, los acuíferos kársticos del Pirineo Aragonés fueron agrupados hacia finales de la década de 1970 por el IGME en los sistemas hidrogeológicos 67 (Sinclinal de Jaca) y 68 (Sinclinal de Tremp). Posteriormente, dichos sistemas dieron lugar a los denominados dominios hidrogeológicos, los cuales se subdividían a su vez en unidades hidrogeológicas. EI PNOMP pertenece a la denominada unidad hidrogeológica 205 (Dominio Pirenaico del Sinclinal de Jaca-Pamplona). Más recientemente, como consecuencia de la Directiva Marco de Agua (2000/60/CE), el PNOMP se incluyó en la masa de agua subterránea 09032 (SierraTendeñeraMonte Perdido).

Las calizas, dolomías y calcarenitas del Cretácico superior e inferior y del Paleoceno-Eoceno constituyen los afloramientos permeables más importantes. En ellos se encuentran los manantiales y las zonas de descarga de agua subterránea más significativas. Los principales sistemas kársticos están ubicados en los materiales del Paleoceno-Eoceno (CHE, 1998; RíosAragüés, 2003; Lambán et al., 2015), entre los que destacan por su desarrollo los sistemas Arañonera, Punta de las Olas y Fuentes de Escuaín. La potencia del conjunto es de 200 a 300 m, aunque el espesor de dichas formaciones aumenta en el sector septentrional por causas tectónicas (pliegues apretados y apilamientos tectónicos), lo que permite el desarrollo de sistemas kársticos en los que se superan los $1000 \mathrm{~m}$ de potencia de zona vadosa (no saturada). El yacente está constituido por las facies terrígenas del Maestrichtiense, responsables de los numerosos manantiales de la red de drenaje que existen en los valles de Añisclo y Ordesa. A techo se emplaza una potente serie margosa del Ilerdiense-Cuisiense (Formaciones Millaris, Meltis y Yeba).

\section{Investigaciones hidrogeológicas}

Dada la importancia de las aguas subterráneas en el PNOMP, el IGME ha iniciado en los últimos años distintas investigaciones hidrogeológicas en el marco del proyecto "Análisis del funcionamiento hidrogeológico de humedales dependientes del agua subterránea" (2013-2016 Ref. IGME 2316)". Para ello se han realizado campañas periódicas de muestreo químico e isotópico de manantiales, además de instalar estaciones para el muestreo de la precipitación (Iluvia y/o nieve) y una estación automática de aforos en la desembocadura del Río Arazas (Valle de Ordesa).

Para el muestreo de la precipitación se han instalado dos estaciones (Figura 3), una junto al Refugio de Góriz, a 2200 m snm y otra en el antiguo Centro de Visitantes del Parador de Ordesa, a $1250 \mathrm{~m}$ snm. Se toman muestras de precipitación acumulada de periodos comprendidos entre uno y tres meses, evitando el fraccionamiento isotópico por evaporación mediante una capa flotante de parafina. En ambas estaciones se toman datos de la precipitación y se muestrea la lluvia y la deposición atmosférica total (húmeda y seca asociada al polvo atmosférico). En las muestras se analizan los iones mayoritarios y el $\delta^{18} \mathrm{O}$ $y$ el $\delta^{2} \mathrm{H}$ del agua.

Se ha instalado una estación de aforos en el Puente de la Ereta, en el río Arazas, antes de su confluencia con el Río Ara (Figura 4). La estación de aforos tiene un sistema de medida de nivel por radar por impulsos de baja energía justo debajo del puente. EI equipo está protegido y configurado para realizar automáticamente una medida del caudal del río cada hora y el envío telemático de estas medidas a la base de datos, donde se recoge esta información. El seguimiento de los aforos está siendo realizado por el Servicio de Hidrología y Aforos de la Confederación Hidrográfica del Ebro (CHE). 

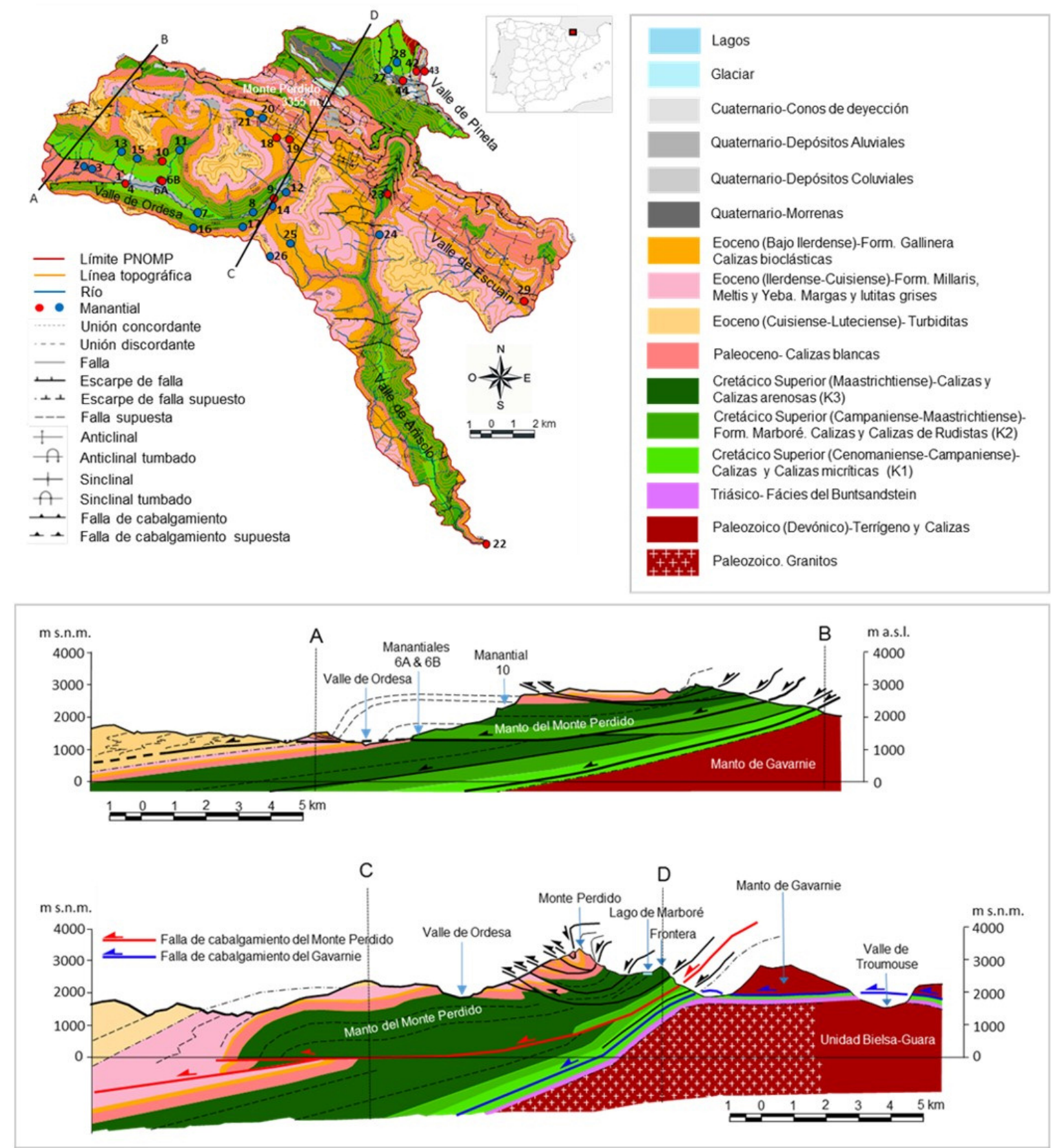

Figura 2. Situación, mapa geológico y cortes geológicos A-B y C-D (modificada de Lambán et al., 2015). Los círculos en rojo indican los manantiales muestreados periódicamente. Los códigos corresponden a los de la Tabla 1.

Figure 2. Situation, geological map and geological cross-sections A-B y C-D (modified from Lambán et al., 2015). The circles in red show the springs sampled periodically. The codes correspond to those in Table 1.

\section{Estimación y distribución espacial de la capacidad de infiltración}

Para evaluar la distribución de la capacidad de infiltración en el PNOMP se ha aplicado una modificación del método APLIS. El método APLIS (Andreo et al., 2008) es un método específico para la evaluación de la capacidad de infiltración en acuíferos carbonatados a partir de las siguientes variables intrínsecas del territorio: altitud $(A)$, pendiente $(P)$, litología $(L)$, zonas 

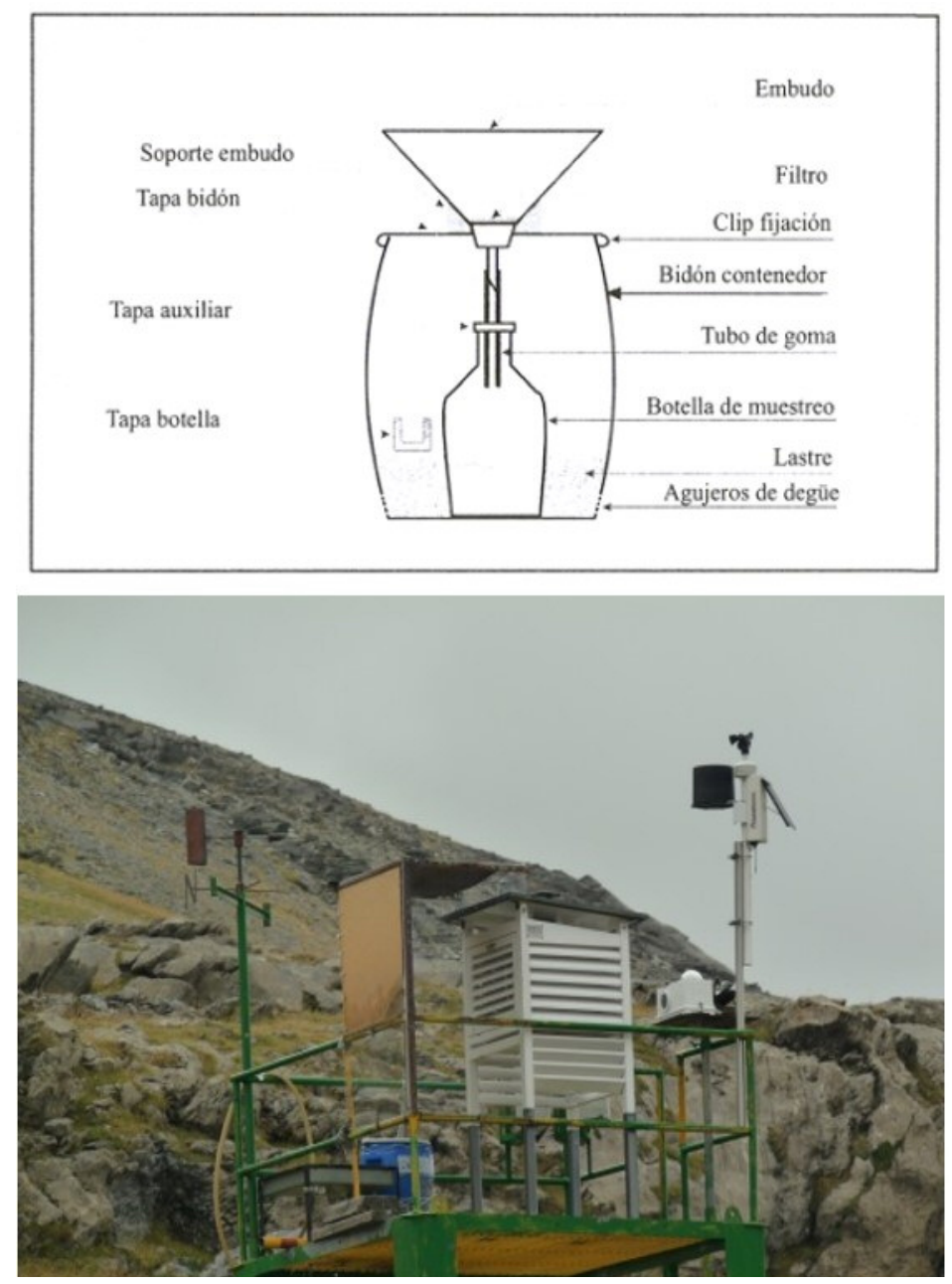
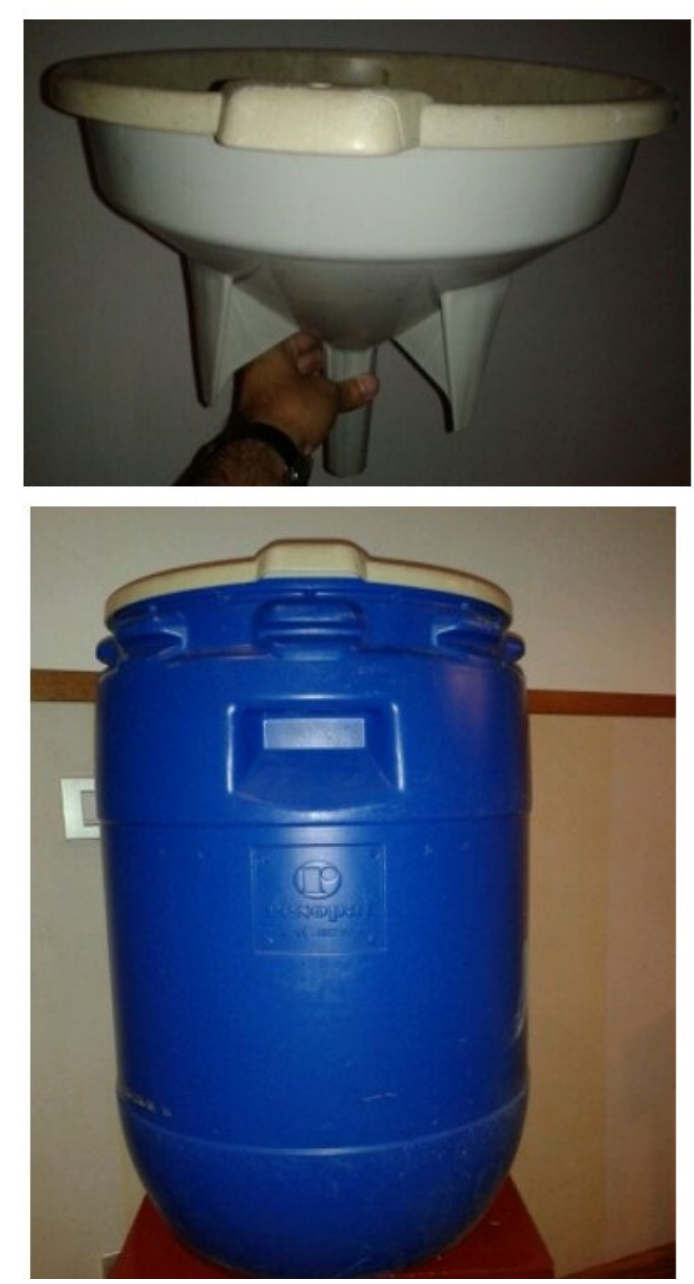

Figura 3. Croquis y componentes de las estaciones instaladas en el PNOMP para el muestreo de la precipitación (lluvia y/o nieve). Figure 3. Drawing and components of the stations installed in the PNOMP for sampling of precipitation (rain and snow).

de infiltración preferencial (/) y suelos (S). Mediante Sistemas de Información Geográfica (SIG), a cada capa de información se le asigna una clasificación según estas variables, con valores entre 1 (mínima participación en la infiltración) y 10 (máxima participación en la infiltración), para luego combinar estas capas según la ecuación: $C l=(A+P+3 L+2 /+S) / 0.9$ y obtener así el mapa de distribución espacial de la capacidad de infiltración $\mathrm{Cl}$.

Para su aplicación en el PNOMP, la altitud y la pendiente se han obtenido mediante el Modelo Digital de Elevaciones (MDE) del Instituto Geográfico Nacional (IGN), la litología a partir de la cartografía geológica realizada recientemente por el IGME a escala 1:25.000, la infiltración preferencial a partir de la cartografía geomorfológica facilitada por la Dirección del PNOMP y un mosaico de ortofotos del Plan Nacional de Ortofotografía Aérea (PNOA) del IGN y los tipos de suelo a partir de un mapa de vegetación facilitado por la Dirección del PNOMP.

La altitud $(A)$ se ha dividido en intervalos de 300 metros $(300 \mathrm{~m} \leq \mathrm{A} \leq 2700 \mathrm{~m} \mathrm{snm})$ y la pendiente $(P)$ en intervalos irregulares $(3 \% \leq P \leq 100 \%)$. La notable carga subjetiva asociada a las variables litología $(L)$ e infiltración preferencial (I) ha llevado a modificar ligeramente las categorías y asignación de pesos establecidos inicialmente por el método APLIS, adaptándolas al PNOMP. Para los suelos (S) se ha hecho una aproximación, clasificando la vegetación en función del tipo de suelo al que se la puede asociar, en base a la clasificación y definición de suelos del IGN.

La aplicación del método APLIS se ha realizado mediante el software ArcGIS en su versión 10.2.2 de ESRI, obteniendo la cartografía de la distribución espacial de la capacidad de infiltración en el PNOMP (Figura 5A). No obstante, hay que tener en cuenta que 

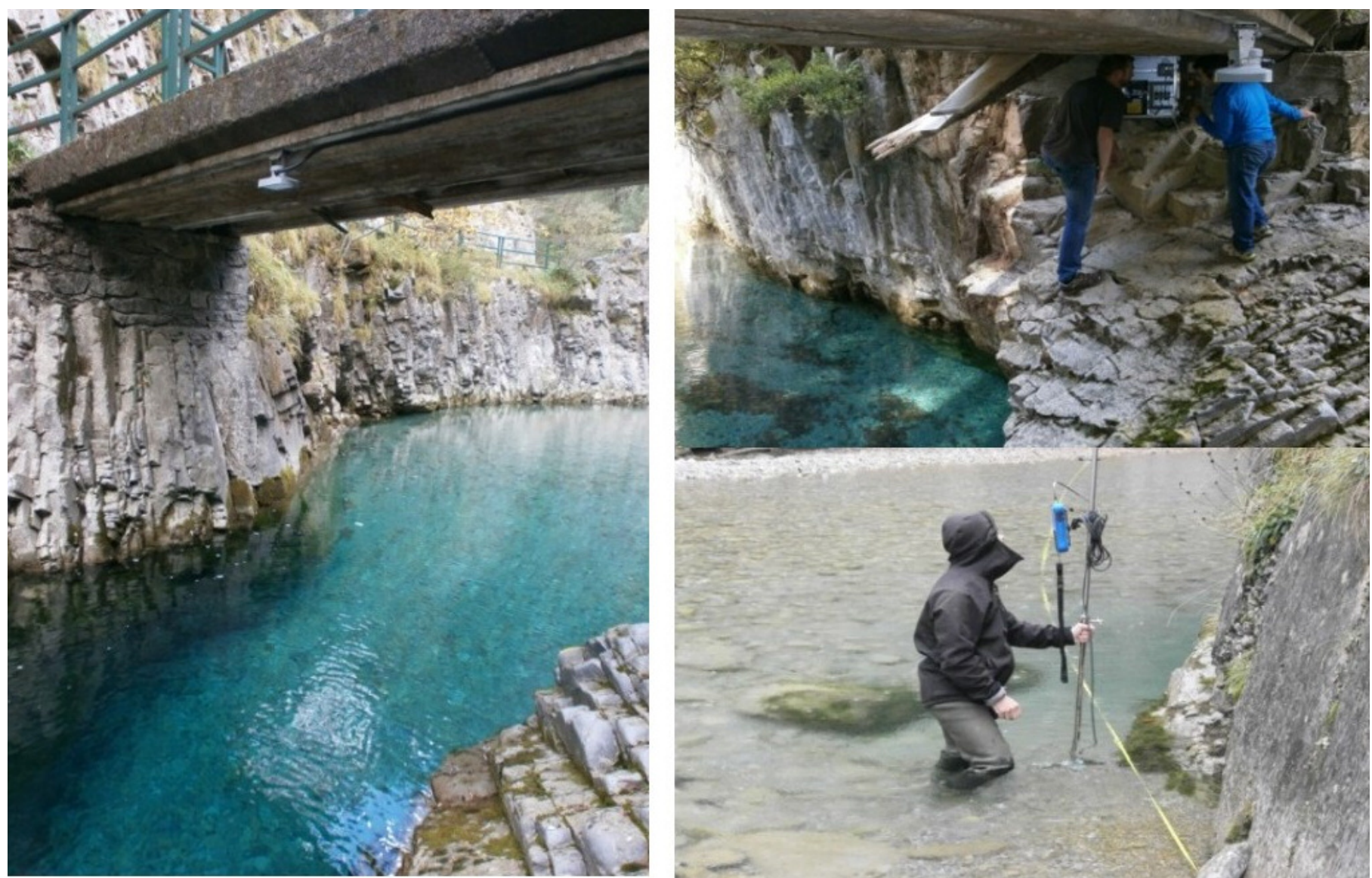

Figura 4. Estación de aforos en el puente de La Ereta (Río Arazas). Figure 4. Gauging station on the La Ereta bridge (Río Arazas).

el método APLIS tiene las siguientes limitaciones y/o incertidumbres: 1) la altitud es un factor poco relevante en la evaluación de la capacidad de infiltración del terreno, 2) en la clasificación y asignación de pesos realizada para la litología se tienen en cuenta factores como la fracturación y karstificación, que también influyen en la capa de infiltración y 3) la importante carga subjetiva al asignar los pesos a las diferentes variables. El método aplicado no permite obtener la recarga ni la tasa media de recarga sino su valor relativo. Para cuantificar hay que considerar la precipitación y algunos valores absolutos de la recarga.

\section{Evaluación preliminar de la recarga}

Se ha realizado una estimación de la recarga en las calizas, dolomías y calcarenitas del PaleocenoEoceno mediante la aplicación de los códigos numéricos HBV y Visual Balan.

En el área de estudio hay datos meteorológicos de tres estaciones pertenecientes a la Agencia Estatal de Meteorología (AEMET) (Figura 1, Tabla 1): 1) Estación de Torla (código 9814), situada a 1053 m snm, con datos de precipitación y temperatura en el intervalo Jul 1953-Ene 2013; actualmente está fuera de servicio, 2) Estación de Fanlo-Góriz (código 9812E), situada a $2195 \mathrm{~m} \mathrm{snm}$, con datos de precipitación y temperatura en el intervalo Ene 1981-Sep 2015; esta estación está gestionada por la Federación Aragonesa de Montaña y 3) Estación de Ordesa-Pradera (código 9812I), situada a $2195 \mathrm{~m} \mathrm{snm}$, con datos de precipitación y temperatura en el intervalo Nov 2014-Sep 2015. Para Góriz se ha generado una serie diaria de precipitación y temperatura que cubre el intervalo Jul 1953Sep 2015, utilizando las series transformadas por anamorfosis gaussiana (Goovaerts, 1997) de las estaciones de Torla y Ordesa-Pradera respecto la serie temporal de Fanlo-Góriz. Estas series han servido para completar los huecos en las series de precipitación y temperatura existentes en las series de FanloGóriz. 
A

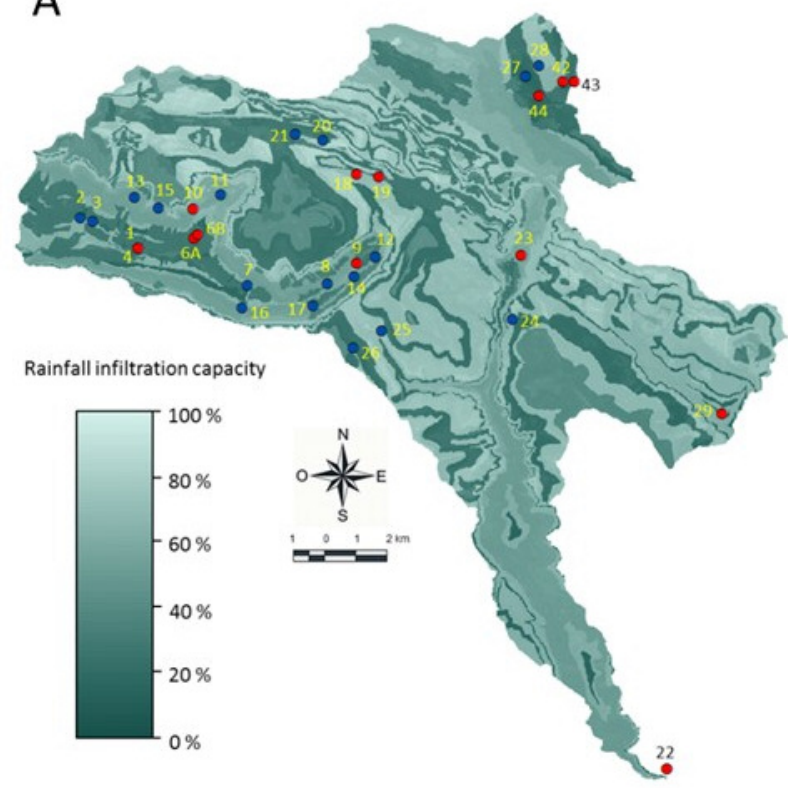

B

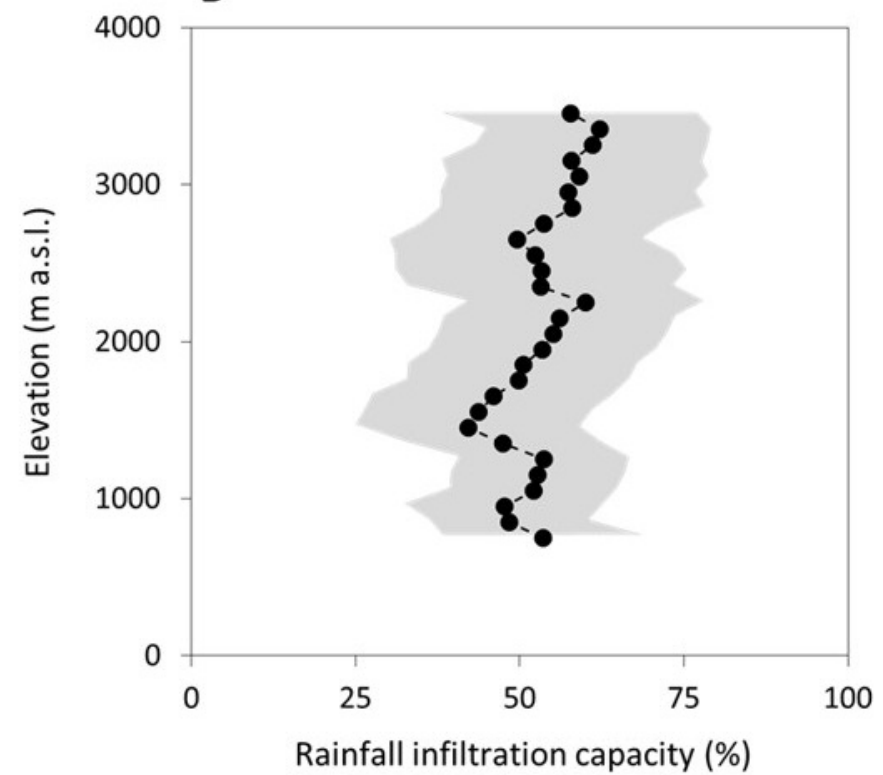

Figura 5. (A) Capacidad de infiltración (Cl) en el PMOMP. Los círculos indican los manantiales; en rojo los muestreados periódicamente. Los códigos corresponden a los de la Tabla 1. (B) Relación entre la cota topográfica y el valor de Cl promediado por intervalos de cota de $100 \mathrm{~m}$. El área sombreada a cada lado del valor medio corresponde a la desviación estándar de la Cl para el intervalo de elevación (tomado de Polo, 2015).

Figure 5. (A) Infiltration capacity $(\mathrm{Cl})$ in the PMOMP. Circles show the springs; in red those sampled periodically. Codes correspond to those in Table 1. (B) Relationship between elevation and averaged $\mathrm{Cl}$ value by altitude intervals of $100 \mathrm{~m}$. The shaded area at each side of the average value corresponds to the standard deviation of $\mathrm{Cl}$ for the elevation interval (from Polo, 2015).

Para la calibración de los resultados se han utilizado los niveles medidos entre septiembre de 2005 y septiembre de 2015 en el piezómetro de Torla (código 09.205.02), perteneciente a la red de control de la CHE.

El código HBV es un modelo agregado de precipitación-escorrentía ampliamente empleado en previsiones hidrológicas y en estudios de balance hídrico (Figura 6). El modelo simula a escala diaria los procesos más importantes de generación de escorrentía, resolviendo el balance de agua en el suelo. Para ello emplea una estructura típicamente de tres cajas (dos del suelo y una del acuífero) y un pequeño número de parámetros agregados (Seibert, 1997, 2005), los cuales se pueden calibrar automáticamente. El código HBV consta de los módulos principales de: 1) fusión y acumulación de nieve, 2 ) humedad del suelo y precipitación efectiva y 3) evapotranspiración. Como resultado del balance de agua se obtiene tanto el caudal de transferencia entre las cajas del modelo como la escorrentía asociada a cada caja del mismo.

El código Visual Balan (Samper et al., 2005), bien conocido y experimentado, es otro modelo agregado que calcula el balance diario de agua en el suelo según el esquema de la Figura 6. Los valores estima- dos de la recarga se pueden calibrar con niveles piezométricos y/o con datos de aforo de manantiales y ríos; además es posible realizar análisis de sensibilidad de los parámetros hidráulicos que intervienen en el proceso de recarga. Es un modelo de acceso público, aplicable a cuencas de todo tipo de tamaños, cuando los parámetros agregados son representativos. Las cuencas heterogéneas se pueden subdividir en un número determinado de subcuencas que tengan características homogéneas en cuanto a clima, uso del suelo, tipo de suelo y gestión hídrica. Permite considerar la zona no saturada como un depósito en el que entra el excedente de agua del suelo (recarga en tránsito) y sale la recarga al medio saturado, con posible escorrentía interna, por ejemplo por niveles colgados considerados de forma agregada.

La aplicación del código HBV (Figura 7; Tablas 2 y 4) muestra que la recarga aumenta según lo hace el valor del $P E R C$, que es el valor límite superior (dintel) de percolación a la celda subterránea. La recarga obtenida corresponde en promedio al $80 \%$ de la precipitación y varía entre el $59 \%$ y el $93 \%$ para valores de PERC entre 5 y $30 \mathrm{~mm} / \mathrm{d}$, respectivamente. Estos valores se comparan con los niveles observados aplicando una función de agotamiento de la reserva sub- 


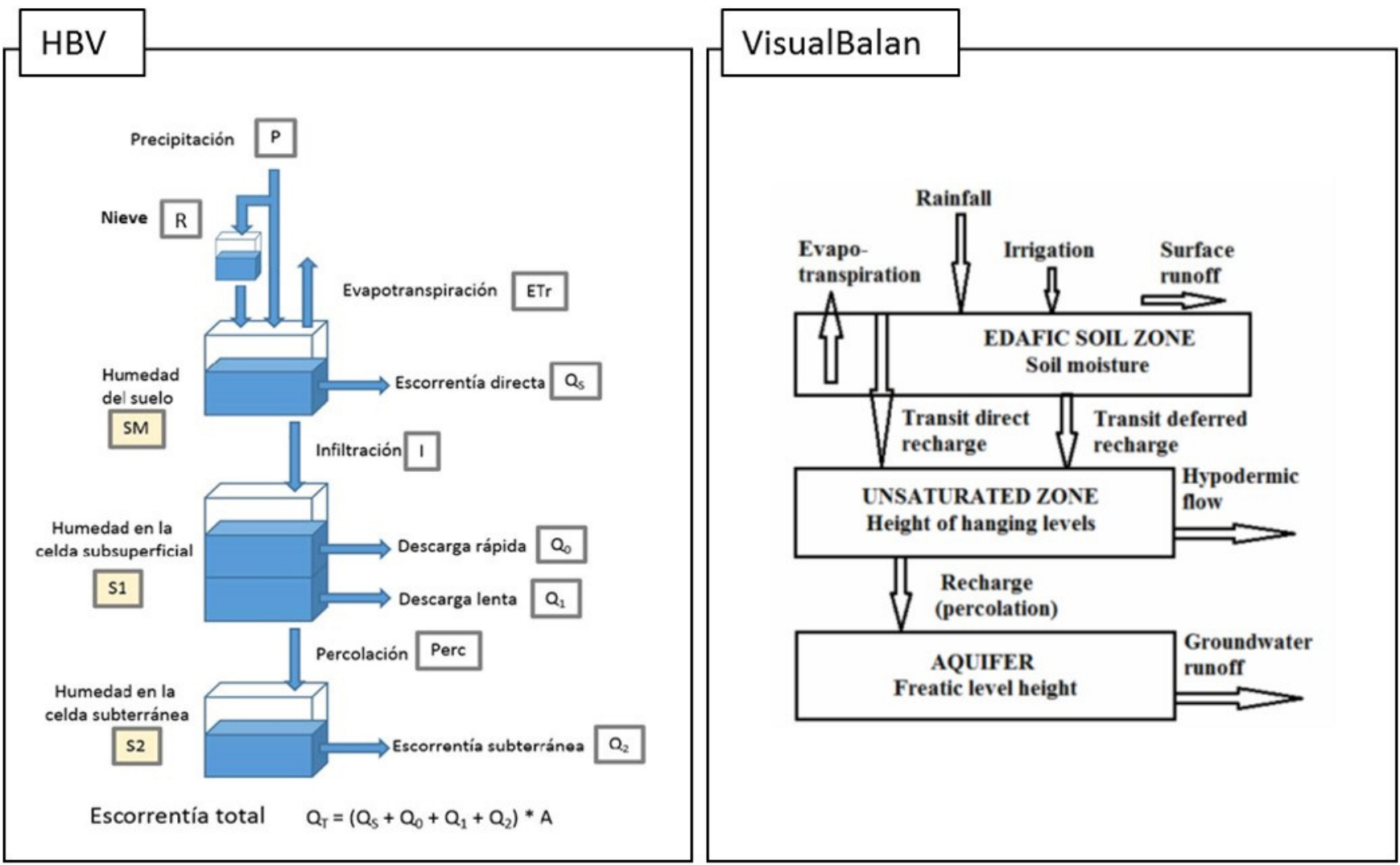

Figura 6. Esquema de los principales componentes del balance de agua en los códigos HBV (Seibert, 2005) y Visual Balan (Samper et al., 2005).

Figure 6. Schematic representation of the main water balance components of HBV (Seibert, 2005) and Visual Balan (Samper et al., 2005) codes.

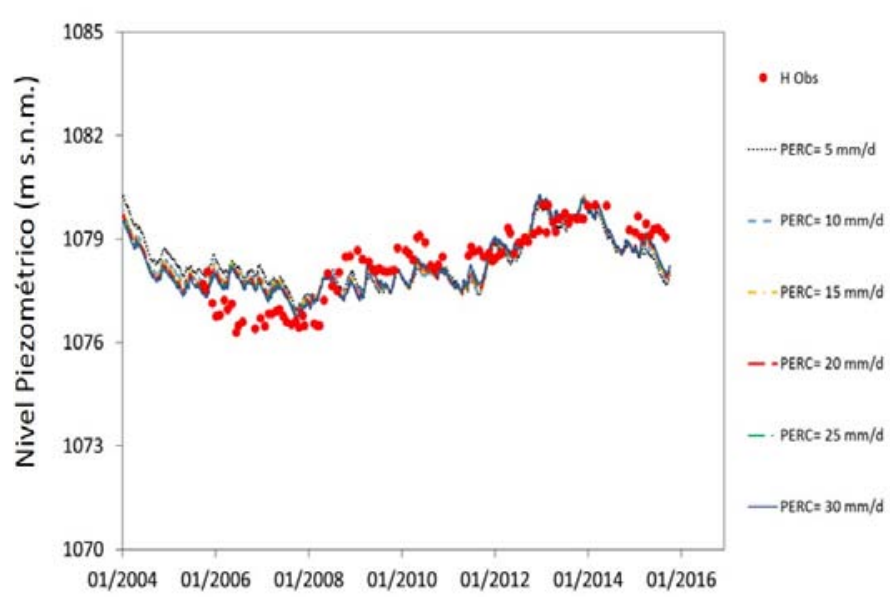

Figura 7. Niveles piezométricos medidos en el piezómetro de Bujaruelo y niveles piezométricos calculados con los valores de recarga según el código HBV, para diferentes valores del parámetro $P E R C$, aplicando los valores de recarga a una celda de agotamiento exponencial.

Figure 7. Groundwater heads measured in the Bujaruelo piezometer and piezometric levels calculated with the recharge values after the HBV code, for different values of the parameter PERC, applied to an exponential recession cell. terránea. En la Figura 7 se puede observar que la respuesta del modelo en función del valor del parámetro $P E R C$ es similar en todos los casos. El coeficiente de almacenamiento es de 0.27 en promedio y varía entre 0.17 y 0.33 para valores de $P E R C$ comprendidos entre 5 y $30 \mathrm{~mm} / \mathrm{d}$.

Para la aplicación del código Visual Balan (Figura 8; Tablas 3 y 4), la ETP se ha calculado mediante el método de Thornthwaite (sólo se dispone de la temperatura) y la ETR aplicando el método lineal. La escorrentía superficial se calcula mediante la Ley de Horton. Para el cálculo de la recarga se ha considerado el método de la curva logística y que hay flujo preferente por fisuras. Para calcular los niveles piezométricos en el acuífero se ha considerado un modelo de dos celdas, que permite obtener un razonable ajuste preliminar de niveles, dado que el valor obtenido de $S$ parece excesivamente alto y los datos de precipitación utilizados pueden diferir de los que realmente han producido la recarga, en especial en 2007 (Figura 8). Los valores de escorrentía superficial, evapotrans- 


\begin{tabular}{|c|c|c|c|}
\hline & \multicolumn{3}{|c|}{ Parámetros } \\
\hline & General & $\begin{array}{c}\text { Zona } \\
\text { vegetation } 1\end{array}$ & $\begin{array}{c}\text { Zona } \\
\text { vegetation } 2\end{array}$ \\
\hline $\operatorname{PERC}(\mathrm{mm})$ & 15 & & \\
\hline$U Z L(\mathrm{~mm})$ & 205 & & \\
\hline$K_{0}\left(\right.$ día $\left.^{-1}\right)$ & 0.60 & & \\
\hline$K_{1}\left(\right.$ día $\left.^{-1}\right)$ & 0.50 & & \\
\hline$K_{2}\left(\right.$ día $\left.^{-1}\right)$ & $810^{-4}$ & & \\
\hline MAXBAS (-) & 7.00 & & \\
\hline PCALT (\%/100m) & 8.76 & & \\
\hline $\operatorname{TCALT}\left({ }^{\circ} \mathrm{C} / 100 \mathrm{~m}\right)$ & -0.55 & & \\
\hline $\operatorname{TT}\left({ }^{\circ} \mathrm{C}\right)$ & & 1.00 & 1.00 \\
\hline CFMAX $\left(\mathrm{mm} /\left(^{\circ} \mathrm{C} \cdot\right.\right.$ día $\left.)\right)$ & & 2.00 & 2,0 \\
\hline SFCF (-) & & 0.80 & 0.80 \\
\hline$C F R(-)$ & & 0.15 & 0.05 \\
\hline$C W H(-)$ & & 0.30 & 0.10 \\
\hline$F C(\mathrm{~mm})$ & & 50 & 30 \\
\hline$L P(\mathrm{~mm})$ & & 0.50 & 0.50 \\
\hline$\beta(-)$ & & 0.50 & 0.50 \\
\hline
\end{tabular}

Tabla 2. Parámetros del modelo HBV obtenidos tras la calibración. Los diferentes valores tabulados son: PERC.= percolación máxima desde la caja de suelo a la caja inferior del acuífero; UZL = parámetro umbral; $\mathrm{K}_{0}=$ coeficiente de recesión rápida; $\mathrm{K}_{1}$ = coeficiente de recesión lenta; $K_{2}=$ coeficiente de recesión del flujo de base; MAXBAS = ancho de base de la función de ponderación triangular; PCALT = gradiente vertical de precipitación; TCALT = gradiente vertical de temperatura; TT = Temperatura umbral; CFMAX = factor grado-día; SFCF = factor de corrección a lluvia de las precipitaciones en forma de nieve; CFR = coeficiente de congelación; $\mathrm{CWH}=$ capacidad de la nieve para la retención de agua; FC = almacenamiento máximo de la humedad del suelo; $L P=$ valor de humedad del suelo por encima del cual AET es igual a PET; $\beta=$ contribución relativa a la escorrentía de la lluvia o el deshielo.

Table 2. Parameters of model HBV after calibration. The different values in the table are: PERC = maximum percolation from the upper to the lower groundwater box; $U Z L=$ threshold parameter; $K_{0}=$ fast recession coefficient; $K_{1}=$ slow recession coefficient; $K_{2}=$ base flow recession coefficient; MAXBAS = base width of the triangular weighting function; PCALT = vertical gradient of precipitation; $T C A L T=$ temperature vertical gradient; TT: threshold temperature; CFMAX: degree-day factor; SFCF: snowfall correction factor; CFR = re-freezing coefficient; $\mathrm{CWH}=$ snow capacity for holding water; $F C$ $=$ maximum soil moisture storage; $L P=$ soil moisture value above which $A E T$ equals $P E T ; \beta=$ relative contribution to runoff from rain or snowmelt.

piración real y recarga calculados son similares a los obtenidos con el código HBV (Tabla 4). Las mayores diferencias se obtienen en la distribución del agua excedente del balance en el suelo, es decir, el reparto entre el flujo hipodérmico (en este caso escorrentía

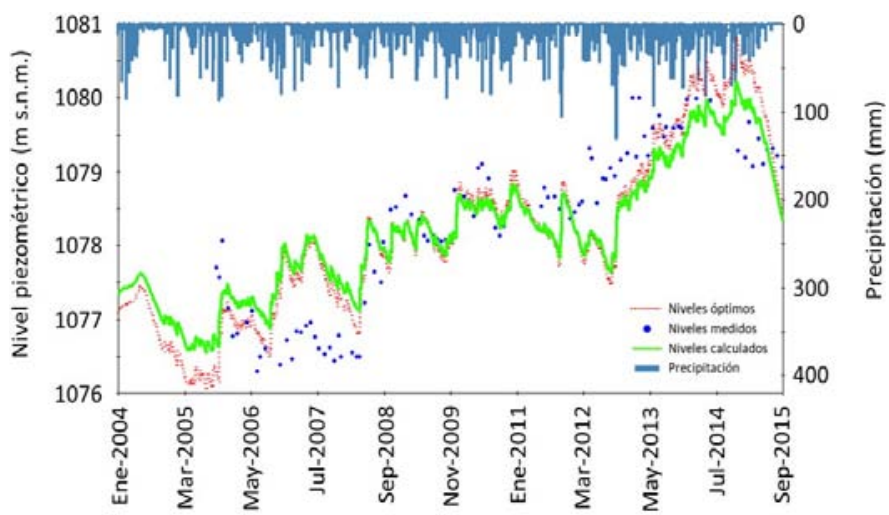

Figura 8. Niveles piezométricos medidos en el piezómetro de Bujaruelo y niveles piezométricos calculados con los valores de recarga según el código Visual Balan.

Figure 8. Observed groundwater levels in the piezometer of Bujaruelo and the corresponding computed values obtained with $B A L A N$ after calibrating the model parameters.

\begin{tabular}{|c|c|c|c|}
\hline \multirow{2}{*}{} & \multicolumn{3}{|c|}{ Parámetros } \\
\hline & Suelo & Zona no saturada & Acuífero \\
\hline$E S P E(\mathrm{~m})$ & 2 & & \\
\hline$C C(\mathrm{~mm})$ & 207 & & \\
\hline$C I M_{0}(\mathrm{~mm})$ & 45 & & \\
\hline$C I M_{1}(\mathrm{~mm})$ & 65 & & \\
\hline$K(\mathrm{~mm} /$ día $)$ & 12.42 & & \\
\hline$\alpha_{\mathrm{h}}(1 /$ días $)$ & & & \\
\hline$\alpha_{\mathrm{p}}(1 /$ días $)$ & & 0.37 & \\
\hline$K_{v}(\mathrm{~mm} /$ día $)$ & & 0.2 & 0.28 \\
\hline$T\left(\mathrm{~m}^{2} /\right.$ día $)$ & & & \\
\hline$S$ & & & \\
\hline
\end{tabular}

Tabla 3. Parámetros del modelo Visual Balan obtenidos tras la calibración. Los diferentes valores tabulados son: ESPE = espesor del suelo; $\mathrm{CC}=$ capacidad de campo; $\mathrm{CIM}_{0}=$ infiltración en el punto de marchitez; $\mathrm{CIM}_{1}=$ infiltración a la capacidad de campo; $\mathrm{K}=$ permeabilidad vertical del suelo; $\alpha_{h}=$ coeficiente de agotamiento del flujo hipodérmico; $\alpha_{p}=$ coeficiente de agotamiento de la percolación; $K_{v}$ $=$ permeabilidad vertical $; \mathrm{T}=$ transmisividad y $\mathrm{S}=$ coeficiente de almacenamiento.

Table 3. Parameters of the Visual Balan model after calibration. The different values in the table are: $E S P E=$ soil thickness; $C C=$ field capacity; $C I M_{0}=$ infiltration at wilting point; $C I M_{1}=$ infiltration at field capacity; $K=$ soil vertical permeability; $\alpha_{h}=$ hipodermic depletion coefficient; $\alpha_{p}=$ percolation depletion coefficient; $K_{v}=$ vertical permeability; $T$ = transmisivity, and $S=$ storage coefficient. 


\begin{tabular}{|c|c|c|c|}
\hline & $\begin{array}{c}\text { Visual } \\
\text { Balan }\end{array}$ & $\begin{array}{c}\text { HBV } \\
\text { (PERC }=\mathbf{5} \mathbf{~ m m} \text { ) }\end{array}$ & $\begin{array}{c}\text { HBV } \\
\text { (PERC =30 } \mathbf{~ m m ) ~}\end{array}$ \\
\hline$E S C O(\%)$ & 0.09 & 0.07 & 0.02 \\
\hline$E T R(\%)$ & 0.21 & 0.18 & 0.18 \\
\hline$I(\%)$ & 0.72 & 0.77 & 0.77 \\
\hline$R E C(\%)$ & 0.45 & 0.59 & 0.93 \\
\hline$F H(\%)$ & 0.27 & 0.34 & 0.05 \\
\hline OAS $(\%)$ & 0.44 & 0.59 & 0.93 \\
\hline
\end{tabular}

Tabla 4. Comparación entre las principales componentes del balance obtenidas mediante la aplicación de los códigos Visual Balan y HBV. Los porcentajes están referidos al valor de la precipitación. $E S C O=$ escorrentía superficial; $E T R=$ evapotranspiración real; $I=$ infiltración (recarga) en HBV y recarga en Visual Balan; $R E C=$ recarga al acuífero; $F H$ = flujo hipodérmico; $Q A S$ = caudal subterráneo. Table 4. Comparison between the main water balance components obtained by the application of the Visual Balan and HBV codes. The percentages refer to precipitation. ESCO = surface runoff; $E T R=$ actual evapotranspiration; I = infiltration (recharge) in HBV and recharge in Visual Balan; $R E C=$ aquifer recharge; $F H=$ interflow; $Q A S=$ groundwater flow .

dentro de la zona no saturada, principalmente como acuíferos colgados temporales) y la recarga al medio saturado. El coeficiente de almacenamiento obtenido (0.28) es muy similar al obtenido aplicando HBV.

La aplicación de los códigos HBV y Visual Balan a partir de los niveles medidos en el piezómetro de Bujaruelo (Torla) permite estimar la escorrentía superficial (ESCO), evapotranspiración real $(E T R)$ y recarga ( $/$ ) para el acuífero Paleoceno-Eoceno del Parque Nacional de Ordesa y Monte Perdido (PNOMP) (Tabla 4).

Los valores obtenidos de ESCO, ETR e I son de 160,360 y 1227 mm/año, respectivamente. Estos valores representan aproximadamente el $10 \%, 20 \%$ y $70 \%$ de la precipitación media anual. Estos resultados son coherentes con los valores esperables para un acuífero (1) desarrollado en calizas con un importante proceso de karstificación, tanto en superficie como en profundidad, (2) donde las mayores precipitaciones se registran entre los meses de otoño y primavera en forma de nieve porosa y (3) donde la nieve cubre durante esos meses la superficie del PNOMP y controla el funcionamiento hidrológico del sistema, como lo muestra el contenido isotópico del agua en los manantiales de la zona (Lambán et al., 2015; Jódar et al., 2016b).

La fusión de la nieve provee un caudal de infiltración permanente, el cual no está limitado por la capacidad de infiltración del terreno dado el alto grado de karstificación en superficie. La presencia del manto de nieve durante varios meses favorece la infiltración del agua de pequeños episodios de lluvia, sin que esta se evapotranspire. Sin embargo la nieve caída, puede disminuir por sublimación antes de fundirse y en espacial por ablación por el viento.

En ambos casos, el valor de la recarga es elevado (i.e. $80 \%$ según HBV y $70 \%$ según Visual Balan), lo que pone de manifiesto la elevada vulnerabilidad de los recursos de agua del parque ante la reducción de recarga ante un posible cambio climático y global y el riesgo que entraña en cuanto a la conservación de parte de sus valores. Las mayores diferencias estriban en cómo se distribuye el agua que abandona el suelo, es decir, el reparto producido entre el flujo de descarga de la zona no saturada (principalmente por niveles colgados) y la recarga. Teniendo en cuenta las características hidrogeológicas del área de estudio y en coherencia con los resultados obtenidos en otras áreas similares, como en los Puertos de Beceite (Espinosa y Custodio, 2014), es de esperar la existencia de flujo por niveles colgados en el PNOMP. Por otro lado, el coeficiente de almacenamiento $S$ obtenido es también similar en ambos casos (entre 0.17 y 0.33 con HBV y 0.28 con Visual Balan), aunque por encima del rango esperable para este tipo de materiales. Esto puede ser debido a un gran desarrollo kárstico, que afecta incluso a fisuras pequeñas, y al incremento de la porosidad de la matriz rocosa.

El periodo de datos piezométricos disponible corresponde a un periodo anormalmente húmedo, el cual se calibra bien, esto es, los valores calculados de la variable de estado del modelo (i.e. caudal de descarga y/o nivel piezométrico) se ajustan a los observados. Esto es en parte una fortaleza de los modelos usados (HBV y Visual Balan), pero para reducir la incertidumbre, tanto en los parámetros calibrados como en los caudales de descarga y/o niveles piezométricos calculados, se requiere un periodo más largo de observación, que considere al menos una parte del comportamiento hidroclimático medio. EI valor de $S$, que se puede aproximar por la porosidad drenable/llenable del acuífero en la zona próxima al nivel freático, puede tener una notable incertidumbre. Si $S$ fuese menor también la recarga sería menor a costa de una mayor descarga lateral desde la zona no saturada del acuífero, mientras que si $S$ aumenta disminuye la recarga $I$, pero la suma $E S C O$ + I varía poco. Esto es algo a seguir investigando con nuevos datos piezométricos y de aforo del caudal de descarga total del sistema acuífero, poniendo énfasis al caudal de base del sistema hidrológico.

Una calibración con datos de aforo hubiera permitido tener una estimación más consistente de las componentes de las descargas superficial, hipodér- 
mica (colgada) y subterránea. En el Anejo I (páginas 234-291) del estudio CHE (2001) se realiza un balance de agua orientativo para la Unidad Hidrogeológica 9.205 (Tendeñera Monte Perdido) en el que se obtiene un caudal total de descarga a cauces (suma de los caudales superficial, hipodérmico y subterráneo) de $36 \mathrm{~L} / \mathrm{s} / \mathrm{km}^{2} / a n ̃ o ~(200 \mathrm{~mm} / a n ̃ o)$, que es muy próximo al

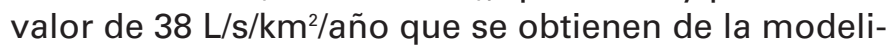
zación realizada con el del Visual Balan (comunicación personal de M.A. García Vera, 2017)

\section{Caracterización hidrogeoquímica e isotópica}

La mayor parte de las muestras de agua analizadas presentan una composición bicarbonatada cálcica o cálcico-magnésica (Figura 9), en coherencia con la naturaleza carbonatada de los principales materiales permeables existentes en el PNOMP. Las aguas bicarbonatadas predominantes se caracterizan por presentar baja conductividad eléctrica (CE), entre 92 y 434 $\mu \mathrm{S} / \mathrm{cm}$, y una temperatura $(\mathrm{T})$ promedio de todos los manantiales muestreados de $10.5^{\circ} \mathrm{C}$.

Hay una buena correlación entre la conductividad eléctrica y el contenido tanto en bicarbonato como en calcio (Figura 10A), lo que unido al predominio de valores de las relaciones $\mathrm{rHCO}_{3} / \mathrm{rCa} \sim 1$ y $\mathrm{rMg} / \mathrm{rCa}<1$
( $r$ significa que la concentración está en meq/L) (Figura $10 \mathrm{~B}$ y $10 \mathrm{C}$, respectivamente) muestra un proceso dominante de disolución de calcita. La relación $\mathrm{rCa} / \mathrm{rSO}_{4}$ (Figura 10D) es mayor que 1 para todas las muestras excepto para Fuen I'Abe [17] y Fuen dero Baño [22], para las que vale 1, lo que apunta a una disolución predominante de anhidrita y/o yeso. Por el contrario, la relación $\mathrm{rMg} / \mathrm{rSO}_{4}$ (Figura 10D) es en general próxima a 1, excepto para los manantiales de Fuen Roya-1 [6A], Fuen Roya-2 [6B] y Fuen dero Baño [22], que presentan mayores contenidos en sulfato $y$ relaciones de $0.67,0.42$ y 0.45 , respectivamente.

En los manantiales muestreados en el Valle de Ordesa se observa un aumento de $\mathrm{CE}, \mathrm{rHCO}_{3}, \mathrm{rCa}$, $\mathrm{rMg}$ y $\mathrm{rMg} / \mathrm{rCa}$ desde cotas altas hacia cotas bajas (Figuras $11 \mathrm{~A}$ a 11C) con una buena correlación $\left(\mathrm{R}^{2}=0.83\right.$ ) entre $\mathrm{rMg}$ y $\mathrm{rMg} / \mathrm{rCa}$ (Figura $11 \mathrm{C}$ ). Por otro lado se observa un aumento de la relación $\mathrm{rCa} / \mathrm{rSO}_{4}$ según aumenta la cota topográfica del manantial muestreado, o sea una correlación inversa entre $\mathrm{rCa} / \mathrm{rSO}_{4}$ y rMg (Figura 11D). Esto podría indicar un proceso de disolución incongruente de dolomita.

A pesar de que en el PNOMP predominan las aguas bicarbonatadas, hay manantiales cuyas características hidrogeoquímicas difieren de la tónica general. Tales son los manantiales de Fuen I Abellana [10] y Fuen Roya-Aguas Abajo [5], con agua sulfatada
A

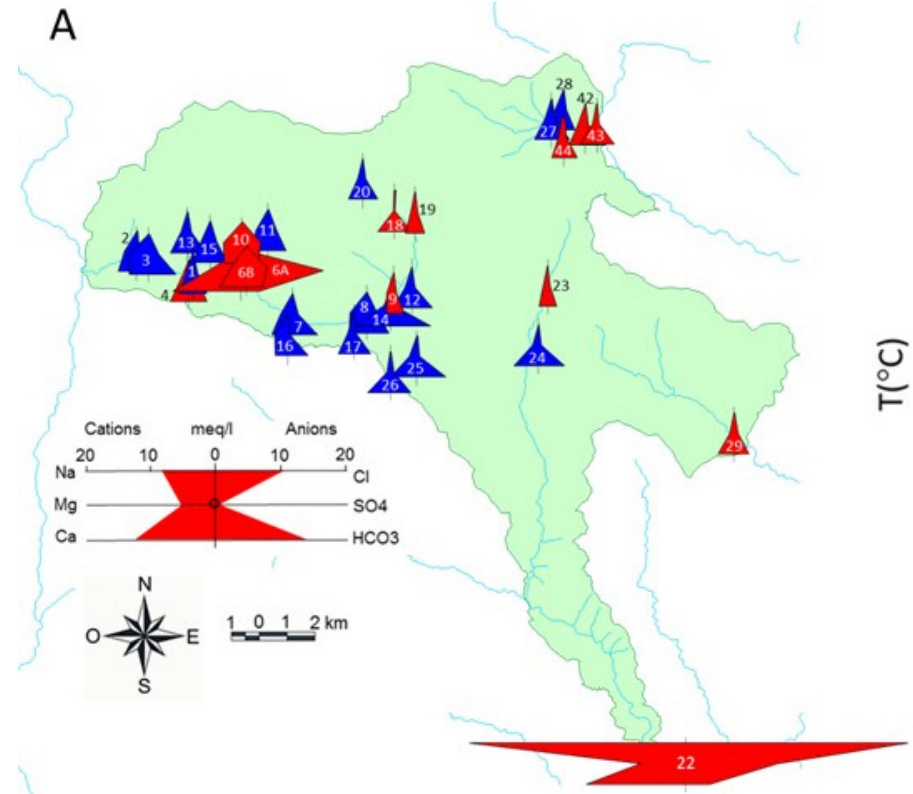

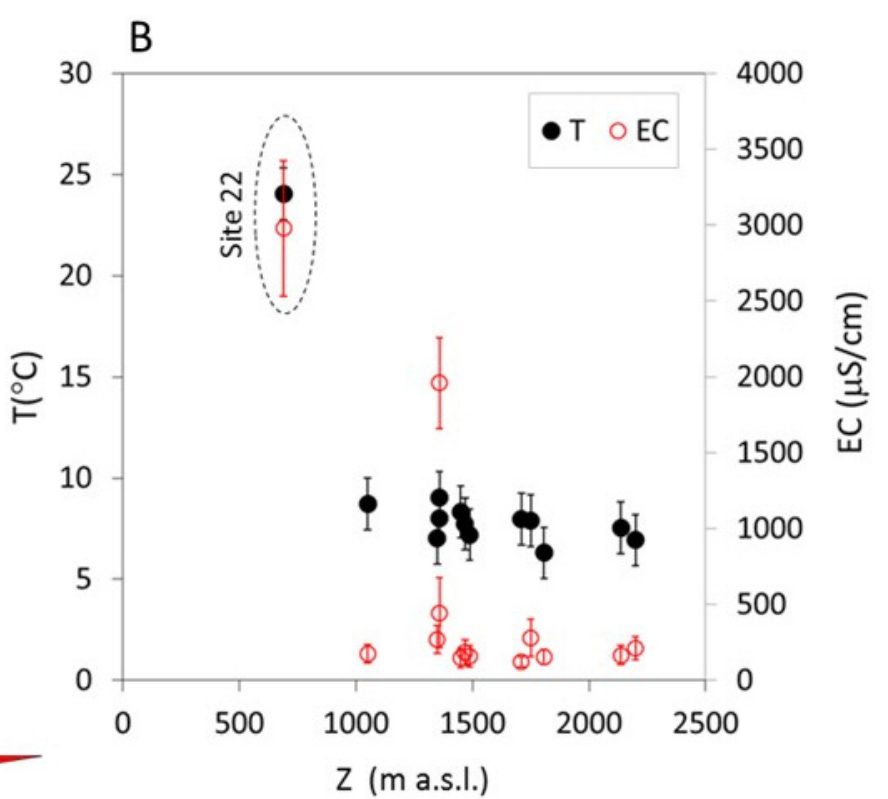

Figura 9. (A) Diagramas de Stiff modificados en los manantiales del PNOMP. Los símbolos en rojo indican los manantiales que se muestrean de manera periódica (B) T (circulo relleno) y CE (circulo vacío) de campo media y desviación estándar. El punto de muestreo [22] corresponde al manantial de Fuen dero Baño.

Figure 9. (A) Modified Stiff diagrams for the PNOMP sprigs. Symbols in red indicate the periodically sampled springs (B) Field T (solid point) y CE (open point) and standard deviation. The sampling site [22] corresponds to Fuen dero Baño spring. 

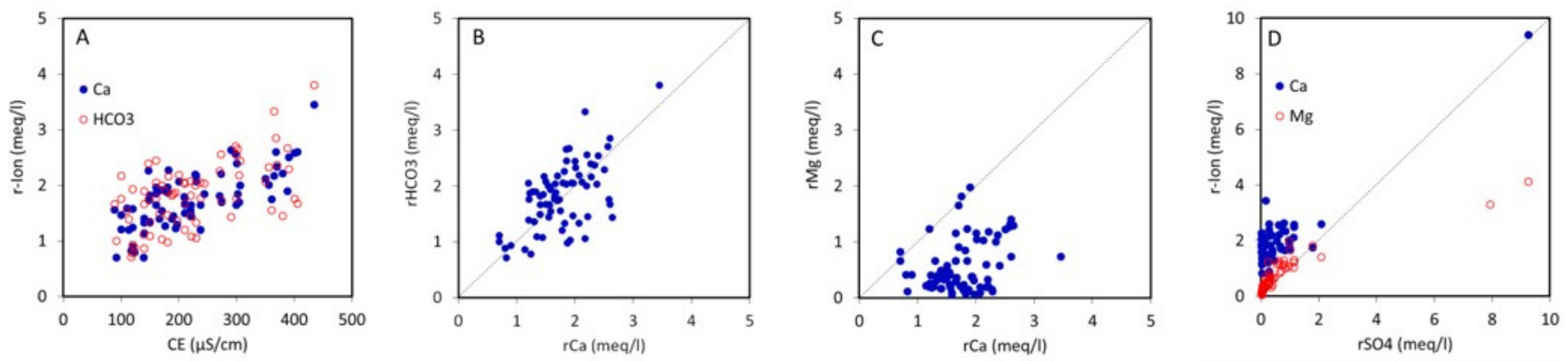

Figura 10. (A) Relación entre $\mathrm{CE}$ y las concentraciones de $\mathrm{HCO}_{3}^{-}$y $\mathrm{Ca}^{2+}$. (B) Relación entre la concentración de $\mathrm{HCO}_{3}{ }^{-}$y $\mathrm{Ca}^{2+}$. (C) Relación entre $\mathrm{Mg}^{2+}$ y $\mathrm{Ca}^{2+}$. (D) Relación entre el sulfato y los iones $\mathrm{Mg}^{2+}$ y $\mathrm{Ca}^{2+}$.

Figure 10. (A) Relationship between $\mathrm{CE}$ and $\mathrm{HCO}_{3}$ y $\mathrm{Ca}^{2+}$ concentrations. (B) Relationship between the la $\mathrm{HCO}_{3}$ y $\mathrm{Ca}^{2+} \mathrm{concentrations}$. (C) Relationship between $\mathrm{Mg}^{2+}$ y $\mathrm{Ca}^{2+}$. (D) Relationship between sulphate and the $\mathrm{Mg}^{2+}$ y $\mathrm{Ca}^{2+}$ ions.
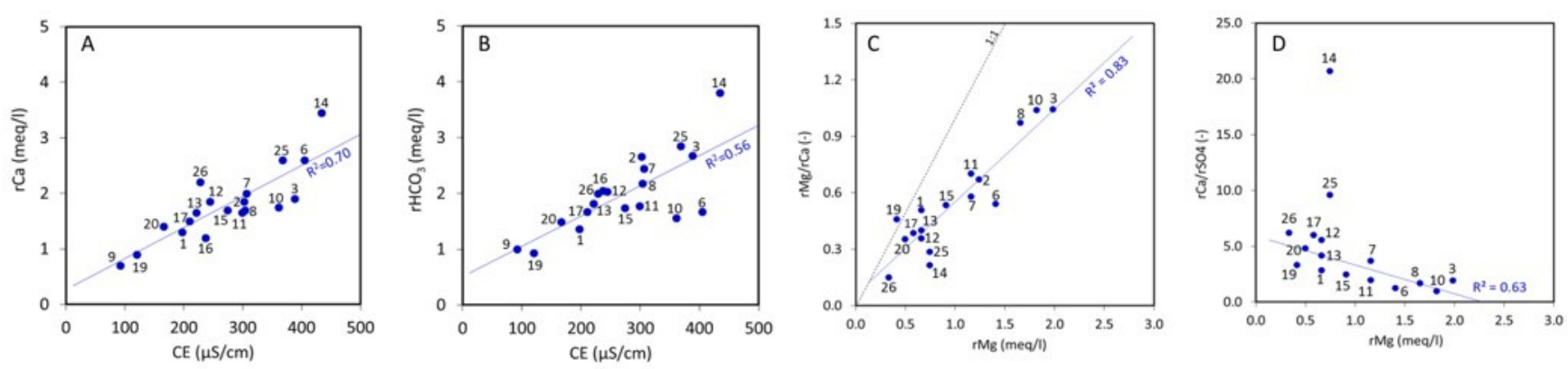

Figura 11. Relaciones hidrogeoquímicas en el ámbito geográfico del valle de Ordesa: (A) $\mathrm{CE}$ y rCa, (B) $\mathrm{CE}_{\text {y }} \mathrm{rHCO}$, (C) $\mathrm{rMg} / \mathrm{rCa}$ y $\mathrm{rMg}$ y (D) $\mathrm{rCa} / \mathrm{rSO}_{4}$ y $\mathrm{rMg}$. La numeración de los puntos se corresponde con la de manantiales de la Figura 1. El prefijo " $\mathrm{r}$ " significa que las concentraciones están en $\mathrm{meq} / \mathrm{L}$.

Figure 11. Hydogeochemical ratios in the geographical area of the Valle de Ordesa: (A) $\mathrm{CE}$ and $r \mathrm{Ca},(\mathrm{B}) \mathrm{CE}$ and $r \mathrm{HCO}{ }_{3},(\mathrm{C}) \mathrm{rMg} / \mathrm{rCa}$ and $r \mathrm{Mg}$ $y(D) r C a / r S$ and $r M g$. Point numbering corresponds that of the springs in Figure 1. The prefix " $r$ " stands for meq/L.

cálcica, y también el manantial termal de Fuen dero Baño [22] (también conocido como Fuente de los Suspiros de Puyarruego), con agua sulfatada-clorurada sódica, T de $26^{\circ} \mathrm{C}$ y CE próxima a $3600 \mu \mathrm{S} / \mathrm{cm}$.

Las precipitaciones que generan la principal fuente de recarga en el PNOMP provienen del Océano Atlántico, como lo demuestra el contenido isotópico estacional promedio de $\delta^{18} \mathrm{O}$ y $\delta^{2} \mathrm{H}$ del agua de lluvia (Figura 12A). Estos valores se encuentran distribuidos sobre la línea meteórica mundial (GMWL), de pendiente 8 y exceso de deuterio de $+10 \%$ (Clark y Fritz, 1997, Gat, 1996). Sin embargo, la composición isotópica del agua subterránea durante el otoño, invierno y primavera muestra un mayor exceso promedio de deuterio, cercano a $+18 \%$ (Figura 12B).

El mayor exceso de deuterio de la precipitación se atribuye al ciclo diario de (1) sublimación diurna de la nieve y evaporación del agua procedente de fusión de nieve que cubre el área de estudio durante gran parte del año y (2) condensación nocturna del vapor de agua atmosférico enriquecido en exceso de deuterio sobre la cobertera nival (Stichler et al., 2001; Froehlich et al., 2008; Christner et al., 2016). El contenido isotópico del agua muestreada en los manantiales tiende a aproximarse a la precipitación de otoño más una contribución de la precipitación de invierno.

El contenido isotópico del agua muestreada, tanto en los pluviómetros como en los manantiales, muestra una clara dependencia estacional (Figura 13). En el caso del agua de lluvia, la amplitud de la variación isotópica estacional aumenta con la cota topográfica de la estación meteorológica donde se realiza el muestreo. Este efecto también se ha observado en las estaciones meteorológicas localizadas a lo largo de un transecto altitudinal a través de los Alpes (Jódar et al., 2016a). En el caso del agua en los manantiales, las diferentes amplitudes están relacionadas con el tiempo de tránsito (Jódar et al., 2016b).

El contenido en tritio en los manantiales muestreados en 2007 varía, en general, entre 6.1×0.6 UT y 

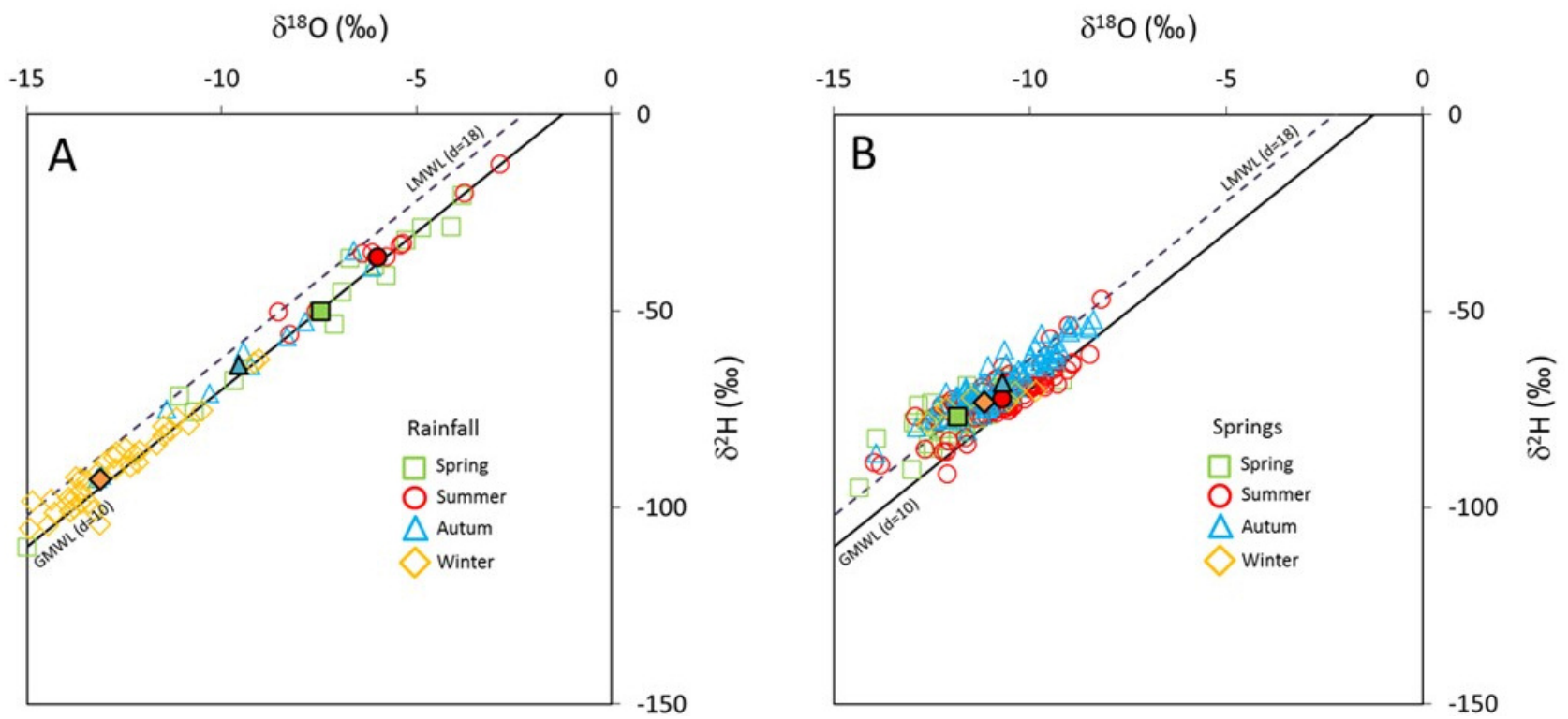

Figura 12. Valores medidos (símbolos sin relleno) y promedios estacionales (símbolo relleno) del $\delta^{18} \mathrm{O}$ y $\delta^{2} \mathrm{H}$ de la precipitación (A) y del agua de los manantiales (B. También se muestran las líneas meteóricas mundial (GMWL) y local (LMWL), teniendo ambas una pendiente de 8 y respectivos excesos de deuterio de $d=10 \%$ y $d=18 \%$.

Figure 12. Average values (open symbols) y seasonal means (solid symbol) of $\delta^{18} \mathrm{O}$ y $\delta^{2} \mathrm{H}$ in precipitation (A) and spring water (B). The World meteorological water line (GMWL) and the local one (LMWL) are also shown; both of them have slope 8 and respective deuteriun excess of $d=10 \%$ and $d=18 \%$.

4.6 \pm 0.6 UT, con un coeficiente de variación de 0.093 . No hay medidas de tritio en el agua de precipitación local, pero los datos de las estaciones REVIP (Red Española de Vigilancia de Isótopos en Precipitación) del CEDEX, muestran que durante los últimos 15 años el contenido medio de tritio prácticamente no ha variado (Díaz-Tejeiro et al., 2009). Además, en las estaciones REVIP más próximas al PNOMP se obtienen valores de tritio similares a los medidos en los manantiales. Esto, junto con las oscilaciones isotópicas estacionales, parece indicar un corto tiempo de tránsito, coherente con la naturaleza kárstica del PNOMP.

\section{Estimación de tiempos medios de tránsito y cota de recarga}

Desde julio de 2007 se viene realizando un muestreo periódico, tanto del agua de la precipitación como la de los manantiales más representativos del PNOMP, los cuales se encuentran distribuidos espacialmente cubriendo un intervalo amplio de cotas de descarga (Figura 1 y Tabla 1).

Las muestras de agua de la precipitación corresponden a acumulaciones mensuales obtenidas en las estaciones del Parador ( 1250 m snm) y Góriz (2200 m snm) (Figura 1 y Tabla 1, P1 y P2). Estas muestras se han tomado para el mismo periodo en el que se han muestreado los manantiales. Los toma-muestras consisten en un embudo de $40 \mathrm{~cm}$ de diámetro de polietileno de baja densidad (LDPE) que captura y guía la precipitación a una botella de LDPE (polietileno de baja densidad) de $5 \mathrm{~L}$ de capacidad, a la que se le añade $350 \mathrm{~mL}$ de aceite de vaselina para que forme una capa flotante que evite la evaporación del agua. Para proteger de la luz solar directa, la botella en la que se recoge la muestra de agua se introduce en un recipiente de LDPE de $30 \mathrm{~L}$ de capacidad.

La estación meteorológica de Fiscal (Figura 1, P3) se encuentra en el pueblo de Borrastre-Fiscal, a $735 \mathrm{~m}$ snm. Esta estación proporciona información sobre el contenido isotópico de la precipitación en las zonas próximas al PNOMP situadas a una cota más baja. Los datos isotópicos corresponden a los eventos individuales de precipitación registrados entre mayo de 2011 y agosto de 2013 (Moreno et al., 2015). Para el cálculo de la media mensual ponderada del contenido isotópico de la precipitación se utiliza la expresión:

$$
\delta_{i}=\frac{\sum_{j=1}^{N_{i}} P_{j} \delta_{j}}{\sum_{j=1}^{N_{i}} P_{j}}
$$

donde $\delta_{i}$ es el contenido isotópico promedio mensual de la precipitación para el i-ésimo mes, $N_{i}$ es el núme- 

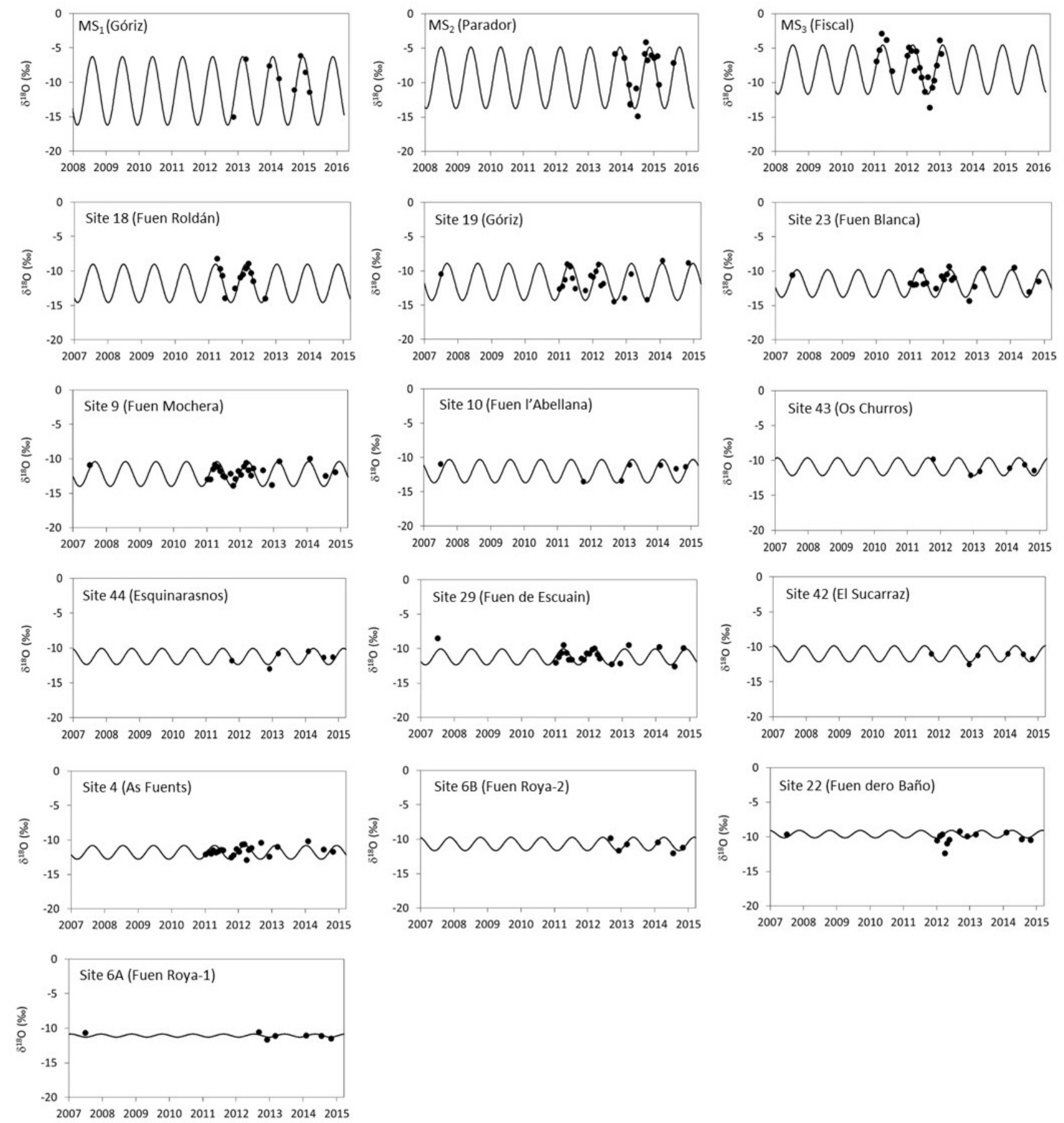

Figura 13. Contenido en $\delta^{18} \mathrm{O}$ observado (círculos) y ajustado (línea) para las muestras de agua procedentes de los pluviómetros (MS $\#$ ) y manantiales (Site \#) muestreados periódicamente en el PNOMP. Los códigos de identificación corresponden a los de la Tabla 1.

Figure 13. Observed $\delta^{18} \mathrm{O}$ content (circles) and adjusted (lines) for water samples from precipitation gauges (MS pled springs (Site \#) in the PNOMP. The identification codes correspond to those in Table 1.

ro de episodios de precipitación y $\delta_{j}$ y $P_{j}$ son el contenido isotópico y el volumen de precipitación del evento $j$-ésimo de precipitación, respectivamente.

Las funciones estacionales de entrada y salida de un trazador se pueden describir mediante el uso de una función sinusoidal (DeWalle et al., 1997; Kabeya et al., 2007; Jódar et al., 2016a, 2016b)

$$
\delta_{\alpha}(t)=A_{\alpha} \sin \left(\omega\left(t-t_{0}\right)+\varphi_{\alpha}\right)+\overline{\delta_{\alpha}}
$$


Luis Javier Lambán, et al., 2019. Investigaciones hidrogeológicas en el Parque Nacional... Boletín Geológico y Minero, 130 (4): 615-640

donde $A$ es la amplitud de la función estacional, $\omega$ es la frecuencia angular, $t$ es el tiempo, $\varphi$ es la fase angular para un tiempo de referencia $t_{o}, \delta$ es el contenido medio de trazador a lo largo de la serie temporal y el subíndice $\alpha$ indica si la función estacional es de entrada $(\alpha=$ in) o salida ( $\alpha=$ out) del sistema hidrológico.

Para una serie temporal de valores medidos de contenido isotópico es posible estimar los parámetros $A, \varphi$ y $\delta$ de manera que la función analítica descrita por la Ecuación 2 se ajuste a los valores del contenido isotópico medido. La estimación de los parámetros se puede hacer fácilmente de manera automática utilizando los programas de optimización que suelen acompañar a las hojas de cálculo comúnmente disponibles, o bien se puede hacer de forma manual.

Para las series temporales de contenido isotópico medidas en las estaciones meteorológicas y los manantiales del PNOMP se han estimado los parámetros $A, \varphi$ y $\delta$ de la Ecuación 2 (Tablas 5 y 6). La Figura 13 muestra el contenido isotópico observado y el ajustado para las estaciones meteorológicas y manantiales.

En el caso de la precipitación, tanto el contenido

\begin{tabular}{|c|c|c|c|}
\hline Código & $\boldsymbol{Z}$ (m snm) & $\boldsymbol{\delta}^{18} \boldsymbol{O}_{\text {in }}(\%)$ & $\boldsymbol{A}_{\text {in }}(\%$ ) \\
\hline P1 & 2200 & -11.25 & 4.97 \\
\hline P2 & 1216 & -9.30 & 4.54 \\
\hline P3 & 780 & -8.10 & 3.58 \\
\hline
\end{tabular}

Tabla 5. Parámetros calibrados de la ecuación 2 aplicado al contenido isotópico estacional del agua de lluvia para las estaciones meteorológicas muestreadas.

Table 5. Calibrated parameters of equation 2 applied to the seasonal isotopic content of rainwater for the sampled meteorological stations.

isotópico promedio $\delta^{18} 0_{\text {in }}$ como la amplitud estacional isotópica $A_{\delta 180 i n}$ muestran una relación lineal con la altitud (Figura 14), con coeficientes de determinación de 0.99 y 0.87 para $\delta^{18} O_{\text {in }}$ y $A_{\text {sisoin, }}$ respectivamente. En general, cuanto más elevada está la estación meteorológica (1) más negativo es el contenido isotópico promedio (agua isotópicamente más ligera) y (2) mayor amplitud estacional isotópica presenta. El gradiente vertical del contenido isotópico promedio de las precipitaciones $\nabla_{z} \delta^{18} O_{\text {in }}$ es de $-2.2 \% \circ / \mathrm{km}$, que es coherente con los valores de los gradientes obtenidos

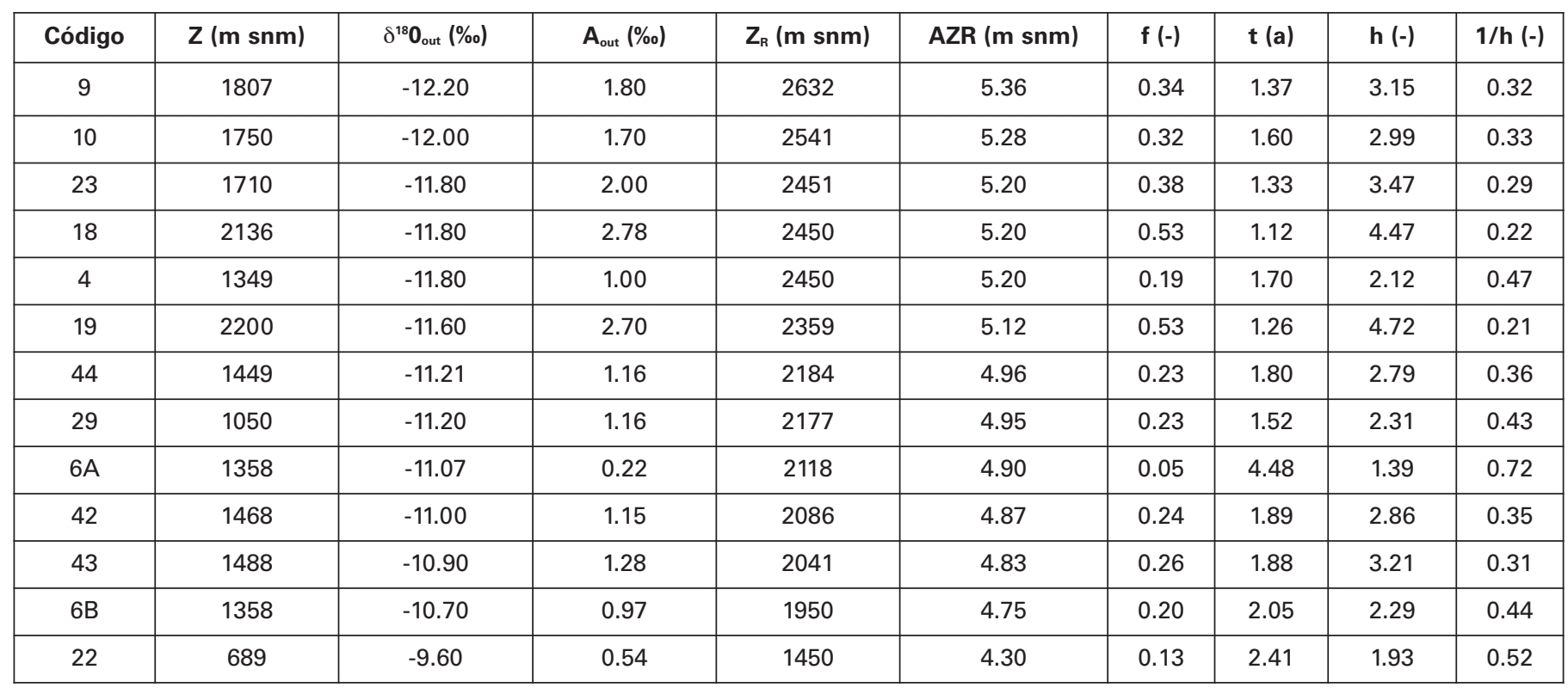

Tabla 6. Parámetros calibrados de la Ecu. 2 aplicados al contenido isotópico estacional en los manantiales del PNOMP. Para cada manantial se proporciona la altitud $\left(Z_{R}\right)$ asociada a su supuesta zona de recarga, la amplitud isotópica estacional asociada a la supuesta zona de recarga $(A Z R)$, el factor de amortiguación (f) que viene dado por el cociente $A_{\text {sin }} / A_{\text {soutr }}$ el tiempo de tránsito $(\tau)$ estimado suponiendo una distribución de tiempos de tránsito correspondiente a un esquema de flujo pistón-exponencial (EPM) y la estimación del parámetro $\eta$ del modelo de flujo EPM.

Table 6. Calibrated parameters of Eq.2 applied to the seasonal isotopic content in springs in the PNOMP. For each spring is given: the altitude associated to the assumed recharge zone $\left(Z_{R}\right)$, the seasonal isotopic amplitude associated to the assumed recharge zone (AZR), the damping factor ( $f$ ) given by the ratio $\mathrm{A}_{\text {sin }} / \mathrm{A}_{\text {sout, }}$ the estimated transit time $(\tau)$ assuming an exponential-piston flow distribution of transit times (EPM), and $\eta$, which is the estimated value of the EPM flow model parameter. 

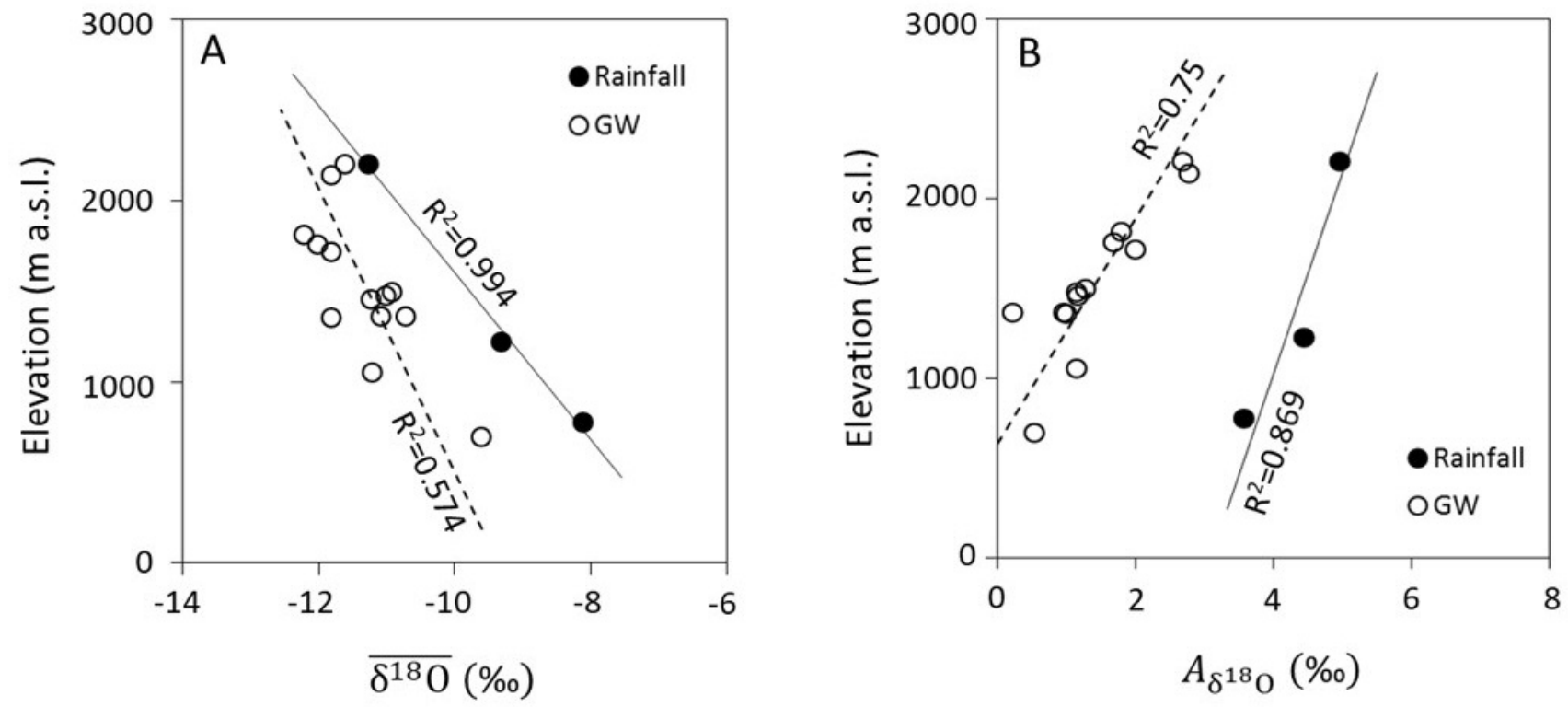

Figura 14. (A) Relación entre $\delta 180$ y la cota topográfica, (B) Relación entre $A \delta 180$ y la cota topográfica. En ambas figuras los datos relativos a la precipitación y los manantiales están marcados con un círculo negro y blanco, respectivamente.

Figura 14. (A) Relationship between $\delta 180$ and elevation, (B) Relationship between A 180 and elevation. In both figures, the data relative to precipitation and springs are shown by black and white dots, respectively.

para diferentes zonas de montaña (Poage y Chamberlain, 2001). El gradiente vertical de la amplitud estacional isotópica de la precipitación $\nabla_{z} A_{\delta 180 i n}$ es de $0.9 \% / \mathrm{km}$, que es similar a la obtenida por Jódar et al., (2016a) para un transecto altitudinal a través de los Alpes. Por otra parte, el hecho de tener una relación constante entre $A_{\delta 180 i n}$ Y la cota topográfica Z pone de relieve la existencia de una fuente común de humedad para las tres estaciones meteorológicas muestreadas en el PNOMP (Jódar et al., 2016b). Este resultado reafirma los resultados obtenidos por Lambán et al. (2014), que usando la relación empírica entre los eventos de precipitación en el PNOMP y su correspondiente patrón de circulación atmosférica a escala sinóptica (Goodess y Palutikof, 1998; Cortesi et al., 2013; Sapriza-Azuri et al., 2013, 2015; Gupta et al., 2016), sugerían que la precipitación en el PNOMP provenía principalmente del Océano Atlántico.

El contenido isotópico promedio en el agua subterránea $\delta_{1800 u t}$ para los manantiales muestreados varía entre $-12.2 \%$ (punto 9) y $-9.6 \%$ o (punto 22) (Tabla 6), mostrando un gradiente vertical medio $\nabla_{z} \delta_{1800 u t}$ de $-1.3 \% / \mathrm{km}$. Este gradiente vertical es similar al reportado por Iribar y Antigüedad (1996) para el Pirineo occidental. $\nabla_{z} \delta_{1800 u t}$ es menor que el valor correspondiente obtenido para la precipitación $\nabla_{z} \delta_{180 i n}$ (Figura 14A), lo que indica que el agua subterránea muestreada en los manantiales proviene de un sistema hidrológico con un patrón de flujo de buena mezcla (Custodio y
Jódar, 2016). En Arce Montejo et al. (2001) se propone un gradiente más elevado a partir del análisis de un muestreo de aguas de todo el Pirineo. La amplitud del contenido isotópico estacional en los $A_{\delta 180 u t}$ varía de $0.19 \%$ o (punto 10 ) a $2.78 \%$ o (punto 18 ) y el correspondiente gradiente vertical de amplitud isotópica estacional $\nabla_{z} A_{\text {sisoout }}$ vale $1.6 \% / \mathrm{km}$, mayor que el obtenido para la precipitación.

El tiempo de tránsito estimado oscila entre 1.12 años (manantial del Fuen Roldán [18]) y 4.48 años (manantial Fuen Roya-1 [6A]), con un valor medio de 1.85 años y una desviación estándar de 0.8 años. Este resultado confirma los cortos tiempos de tránsito obtenidos por Lambán et al. (2015) mediante el análisis del contenido de tritio en el agua muestreada en algunos manantiales del PNOMP. De hecho, la distribución espacial de los tiempos de tránsito obtenida en el presente trabajo es coherente con el modelo conceptual de funcionamiento del sistema acuífero del PNOMP planteado en dicho trabajo. Los manantiales con el tiempo de tránsito más corto (es decir, los puntos de muestreo 18, 19, 23 y 9) corresponden a los manantiales de descarga de los materiales del Eoceno-Paleoceno, más karstificados. Los manantiales con el tiempo de tránsito más largo (es decir, los puntos de muestreo $6 \mathrm{~A}, 6 \mathrm{~B}, 4,42,43$ y 44 ) corresponden a manantiales descargando a partir de los materiales del Cretácico Superior.

El sistema acuífero se comporta como un sistema 
de flujo pistón-exponencial (EPM), una parte con flujo de pistón (PF) y otra con flujo exponencial (EM) conectados en serie y diferente distribución de tiempos de tránsito (Jódar et al, 2014; Zuber, 1986; Małoszewski y Zuber, 1982; Leibundgut et al., 2011). La parte PF sólo retrasa la señal isotópica mientras que la parte EM retrasa y amortigua la señal de entrada del trazador (Małoszewski y Zuber, 1982). La Figura 15A muestra la dependencia obtenida entre $f y \tau$ para los manantiales muestreados. Como se puede ver, cuanto mayor es menor es el factor de amortiguación $f$.

El modelo EPM depende del parámetro $\eta$, que se define como la relación entre del volumen total del sistema y el volumen asociado a la distribución exponencial de los tiempos de tránsito. Los valores de $\eta$ oscilan entre 1 (es decir, modelo de flujo exponencial $E M)$ e infinito. Por lo tanto, $1 / \eta$ oscila entre 0 y 1 . La Figura 15B muestra la relación obtenida entre $1 / \eta$ y $\tau$ para los manantiales muestreados. El volumen asociado a la distribución de los tiempos de tránsito EM aumenta a medida que aumenta el tiempo de tránsito del sistema hidrológico, y viceversa. Este resultado muestra cómo el sistema integra la contribución de las diferentes líneas de flujo cuando se produce la descarga del acuífero. Los manantiales que descargan líneas de flujo de corta longitud se corresponden con los puntos de muestreo con mayor proporción de distribución PF de los tiempos de tránsito y por lo tanto con una menor amortiguación respecto a la fun- ción isotópica estacional de entrada de la precipitación. A medida que el tiempo de tránsito aumenta, también aumenta la longitud de las líneas de flujo. Esto favorece la transición hacia un sistema de mezcla perfecta (EM) en la distribución de los tiempos de tránsito, anulando el papel desempeñado por la parte PF en la respuesta del sistema hidrológico.

Los valores estimados de la cota de recarga $Z_{R}$ para los manantiales muestreados en el PNOMP están comprendidos principalmente entre $1950 \mathrm{~m}$ (manantial Fuen Roya-2 [6B]) y 2632 m snm (manantial de Fuen Mochera [9]), cubriendo un intervalo de elevación de cerca de $700 \mathrm{~m}$. Este resultado es coherente con el obtenido por Polo (2015) usando el método APLIS (Andreo et al., 2008) para determinar la distribución espacial de la capacidad de infiltración en el PNOMP. La Figura 5A muestra la distribución espacial de la capacidad de infiltración de lluvia en el PNOMP. La Figura 5B muestra la variación de la capacidad de infiltración $(\mathrm{Cl})$ del terreno con la cota topográfica. $\mathrm{Cl}$ oscila entre el $40 \%$ y el $60 \%$, mostrando un ligero aumento con la cota; está condicionado por un mayor desarrollo del karst y una menor densidad de vegetación en altura.

La variación vertical de $A_{\text {sizoin }}$ con la cota topográfica se puede utilizar para obtener información adicional sobre los procesos que controlan el funcionamiento del sistema hidrológico. Como se ha visto, la capacidad de infiltración de lluvia es moderada a alta en casi toda la zona de estudio (Figura 5), el tiempo
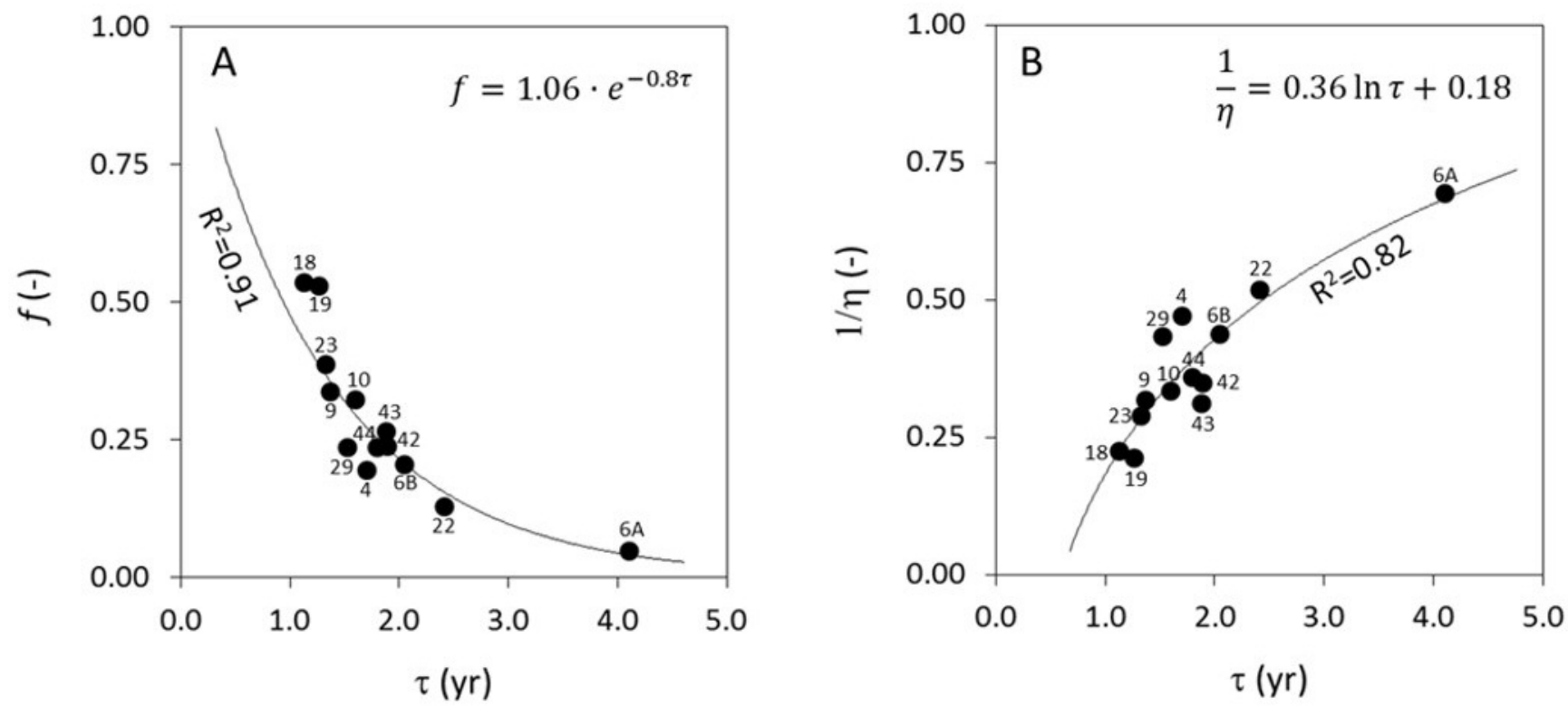

Figura 15. (A) Relación entre $f y \tau$. (B) Relación entre $1 / \eta$ y $\tau$. Los números corresponden a los de la Tabla 1.

Figure 15. (A) Relationship between $f$ and $\tau$. (B) Relationship between $1 / \eta$ and $\tau$. Numbering corresponds to that in Table 1. 
de tránsito del agua subterránea aumenta a lo largo de la línea de flujo (Figura 15A) y el sistema de flujo del agua subterránea tiende hacia un esquema de mezcla perfecta según aumenta su tiempo de tránsito (Figura 15B). Con todo ello cabría esperar que el manantial que presenta el menor valor de la amplitud del contenido isotópico estacional $A_{\text {sizoin }}$ tenga asociado el mayor tiempo de tránsito $\tau$. Sin embargo, esto no ocurre en el caso del manantial de Fuen dero Baño [22]. Una hipótesis que podría explicar este comportamiento anómalo es la existencia en el punto de descarga de un proceso de mezcla entre dos aguas de diferentes orígenes, una de recarga local, con un tiempo de tránsito corto y por ende mostrando una dependencia estacional en el contenido isotópico, y otra de descarga regional, más salina y con mayor temperatura. Por lo tanto, el contenido isotópico del manantial de Fuen dero Baño [22] se puede expresar como una combinación lineal de esas dos aguas. Teniendo en cuenta lo anterior, el contenido isotópico observado en Fuen dero Baño indica una proporción entre el $72 \%$ y el $61 \%$ para el agua de procedencia local y entre el $28 \%$ y el $39 \%$ para la de procedencia regional (Jódar et al., 2016b).

\section{Otras investigaciones en desarrollo}

En la actualidad se están desarrollando otras investigaciones hidrogeológicas en el PNOMP relacionadas con: 1) identificación de otros posibles nuevos acuíferos, 2) caracterización hidrogeoquímica e isotópica de la nieve en función de la altitud de muestreo, 3) caracterización hidrogeoquímica e isotópica de las aguas subterráneas integrando los manantiales muestreados en la cabecera del Valle de Pineta y 4) estudio del papel de las aguas subterráneas en el funcionamiento del Lago de Pineta (o Lago de Marboré). Estas líneas de trabajo ayudarán a caracterizar los procesos que gobiernan el funcionamiento del complejo sistema hidrológico del PNOMP

\section{Conclusiones}

Las investigaciones hidrogeológicas realizadas hasta el momento en el Parque Nacional de Ordesa y Monte Perdido (PNOMP) han permitido obtener una primera distribución espacial de la capacidad de infiltración en todo el parque, así como unos valores de escorrentía superficial, evapotranspiración e infiltración para el acuífero Paleoceno-Eoceno entre el 70 y el $80 \%$ de la precipitación media anual. Dichos valores ponen de manifiesto la elevada vulnerabilidad que presenta el parque y sus recursos de agua ante una reducción de la recarga a causa del posible cambio climático y global, la importancia de la precipitación que favorece una alta humedad en el suelo, la karstificación superficial y en profundidad existente en estos materiales y la presencia de una cubierta de nieve durante varios meses al año que controla el funcionamiento de la recarga y por ende de la descarga del sistema acuífero. Esto conlleva el riesgo de reducción de parte de los valores naturales del Parque.

La química del agua subterránea está controlada por los iones $\mathrm{HCO}_{3}{ }^{-}, \mathrm{Ca}^{2+}$ y $\mathrm{Mg}^{2+}$, en coherencia con las calizas, dolomías y calcarenitas, tanto del Cretácico superior como del Paleoceno-Eoceno inferior. Desde un punto de vista cualitativo, el principal proceso hidrogeoquímico observado es la disolución de calcita. La interpretación de los datos hidrogeoquímicos obtenidos hasta el momento apunta hacia la existencia de (1) un posible proceso de disolución incongruente de dolomita en el Valle de Ordesa, (2) un proceso de disolución de yeso/anhidrita en los manantiales de Fuen Roya-1 y Fuen Roya-2, Fuen l'Abellá y Fuen dero Baño, y (3) un proceso de mezcla con agua procedente de flujos regionales más profundos en Fuen dero Baño.

En la vertiente sur de la zona central del Pirineo se observa una relación lineal entre la amplitud del contenido isotópico estacional de la precipitación y la cota topográfica de la precipitación. Esta relación es constante y confirma que el Océano Atlántico es la principal fuente de humedad para la precipitación en el PNOMP, tal y como también lo sugieren otros autores.

La zona de recarga de los manantiales muestreados se encuentra a una altitud entre 1950 y 2600 m snm. Como la señal isotópica de la precipitación se propaga a través del sistema hidrológico con la recarga, la existencia de un gradiente vertical en la amplitud del contenido isotópico estacional de la precipitación tiene un efecto directo sobre la respuesta del sistema. Por lo tanto, este hecho se debe tener en cuenta cuando el tiempo de tránsito se obtenga como una función de la amortiguación de la amplitud del contenido isotópico estacional de la descarga del sistema hidrológico.

El sistema hidrogeológico presenta tiempos de tránsito cortos, en concordancia con la naturaleza kárstica del parque. A pesar de la pequeña dispersión de los valores de los tiempos de tránsito obtenidos para los manantiales muestreados hasta el momento, se observa que el sistema hidrológico tiende hacia una distribución de esos tiempos de transito según un modelo exponencial de flujo, aumentando la mez- 
cla cuanto mayor el tiempo de tránsito. El origen de la descarga del manantial termal de Fuen dero Baño puede ser explicado como el resultado de una mezcla entre dos componentes: una regional y otra procedente de la recarga local con un tiempo de tránsito relativamente corto.

\section{Agradecimientos}

Esta investigación se ha llevado a cabo en el marco del proyecto CANOA-73.3.00.44.00 "Análisis del funcionamiento hidrogeológico de humedales dependientes del agua subterránea", financiado por el Instituto Geológico y Minero de España (IGME), y en el marco del proyecto "Anillo" ACT-1203 del CONICYT, Chile. Los autores agradecen a la Dirección del Parque Nacional de Ordesa y Monte Perdido, a Elena Villagrasa (Gobierno de Aragón), a Fernando Carmena e Ignacio Gómez (SARGA), a los guardas del refugio de Góriz y a Ana Moreno y Blas Valero (Instituto Pirenaico de Ecología IPE-CSIC) su colaboración. Los datos meteorológicos han sido proporcionados por la Agencia Estatal de Meteorología (AEMET) y los análisis químicos han sido realizados en los laboratorios del Instituto Geológico y Minero de España en Tres Cantos (Madrid) y del Instituto Pirenaico de Ecología en Zaragoza. Los análisis isotópicos han sido realizados en los laboratorios del CETA-CEDEX (Madrid) y IACT-CSIC (Granada). Se agradecen las detalladas y oportunas observaciones aportadas por el Dr. Miguel Ángel García Vera.

\section{Referencias}

Andreo, B., Vías, J.M., Durán, J.J., Jiménez, P., López Geta, J.A., Carrasco, F. (2008). Methodology for groundwater recharge assessment in carbonate aquifers: application to pilot sites in southern Spain. Hydrogeology Journal, 16: 911-925.

Araguás Araguás, L., Froehlich, K., and Rozanski, K. (2000). Deuterium and oxygen 18 isotope composition of precipitation and atmospheric moisture. Hydrological Processes, 14(8): 1341-1355.

Arce Montejo, M., García Vera, M.A., Arqued Esquiá, V.M. (2001). Caracterización del oxígeno 18, deuterio y tritio en las aguas del Pirineo. En: A. Medina y J.Carrera, Las Caras del Agua Subterránea, Eds. IGME, Temas Aguas Subterráneas, I: 387 393. ISBN:84 78404279.

Benito Alonso, J.L. (2006). Vegetación del Parque Nacional de Ordesa y Monte Perdido. Serie Investigación 50. Consejo de Protección de la Naturaleza de Aragón. Zaragoza.

Boronina, A., Renard, P., Balderer, W., Stichler, W. (2005)
Application of tritium in precipitation and in groundwater of the Kouris catchment (Cyprus) for description of the regional groundwater flow. Applied Geochemistry, 20:1292-1308.

Carreras, J., Santanach, P. (1983). El Hercínico de los Pirineos. Geología de España, Libro jubilar Rios, JM, Instituto Geológico y Minero de España, 1, 536-549.

CHE. (1998). Catalogación de los acuíferos de la Cuenca del Ebro. Oficina de Planificación Hidrológica. Confederación Hidrográfica del Ebro, Zaragoza.

CHE. (2001). Estudio de los recursos hidricos subterráneos de los acuíferos de la margen izquierda del Ebro. Zona Oriental. Modificacion $n^{\circ} 1$. Confederación Hidrográfica del Ebro, Zaragoza.

Clark, I., Fritz, P. (1997). Environmental isotopes in hydrogeology. Lewis Publishers, New York: 1-328.

Collins, D.N., Gordon, J.Y. (1981) Meltwater hydrology and hydrochemistry in snow- and ice-covered mountain catchments. Nordic Hydrology, 12:319-334.

Cortesi, N., Trigo, R.M., Gonzalez-Hidalgo, J.C., Ramos, A.M. (2013). Modelling monthly precipitation with circulation weather types for a dense network of stations over Iberia. Hydrology and Earth System Sciences, 17(2): 665678.

Christner, E., Kohler, M., Schneider, M. (2016). The influence of snow sublimation on stable isotopes of water vapor in the atmospheric boundary layer of Central Europe. Atmos. Chem. Phys. Discuss.,

Custodio, E., Jódar, J. (2016). Simple solutions for steady-state diffuse recharge evaluation in sloping homogeneous unconfined aquifers by means of atmospheric tracers. Journal of Hydrology, 540: 287-305.

DeWalle, D.R., Edwards, P.J., Swistock, B.R., Aravena, R., Drimmie, R.J. (1997). Seasonal isotope hydrology of three Appalachian forest catchments. Hydrological Processes, 11(15): 1895-1906.

Díaz-Teijeiro, M.F., Rodríguez-Arévalo, J., Castaño, S. (2009). The Spanish network for surveillance of isotopes in precipitation: spatial isotopic distribution and contribution to the knowledge of the hydrological cycle. Ingeniería Civil, 155: 87-97

Espinosa, S., Custodio, E. (2014). Comparación de la recarga natural estimada con el balance de agua en el suelo y con el balance de la deposición atmosférica de cloruro en un sistema carbonatado en el área del Baix Ebre, Cataluña, España. Ingeniería del Agua, 20: 135-155.

Froehlich, K., Kralik, M., Papesch, W., Rank, D., Scheifinger, H., Stichler, W. (2008). Deuterium excess in precipitation of Alpine regions - moisture recycling. Isotopes in Environmental and Health Studies, 44(1): 1-10.

Gat, J.R. (1996). Oxygen and hydrogen isotopes in the hydrologic cycle. Annual Review of Earth and Planetary Sciences, 24(1): 225-262.

Gonfiantini, R., Roche, M.A., Olivry, J.C., Fontes, J.C., Zuppi, G. M. (2001). The altitude effect on the isotopic composition of tropical rains. Chemical Geology, 181(1): 147-167. https://dx.doi.org/10.1016/S0009-2541(01)00279-0

González-Fernández, B., Meléndez-Asensio, M., MenéndezCasares, E. (2009). Hydrogeological characterization of 
carbonated Jurassic aquifers in the Gijón-Villaviciosa basin (Asturias, N Spain) by means of geochemical and isotopic techniques. Environmental Earth Sciences, 59(4), 913-928.

Goodess, C.M., Palutikof, J.P. (1998). Development of daily rainfall scenarios for southeast Spain using a circulation-type approach to downscaling. International Journal of Climatology, 18: 1051-1083.

Goovaerts, P. (1997). Geostatistics for natural resources evaluation. Oxford University Press, London. 496 pp.

Gremaud, V., Goldscheider, N., Savoy, L., Favre, G., Masson, H. (2009). Geological structure, recharge processes and underground drainage of a glacierised karst aquifer system, Tsanfleuron-Sanetsch, Swiss Alps. Hydrogeology Journal 17: 8. 1833-1848

Gremaud, V., Goldscheider, N. (2010). Geometry and drainage of a retreating glacier overlying and recharging a karst aquifer, Tsanfleuron-Sanetsch, Swiss Alps. Acta Carsologica 39: 2. 289-300.

Gupta, H.V., Sapriza-Azuri, G., Jódar, J., Carrera, J. (2016). Circulation pattern-based assessment of projected climate change for a catchment in Spain. Journal of Hydrology.

Gustafson, J.R. (2007). Snowmelt water isotope fractionation in high elevation seasonal snowpacks: implications for isotope hydrograph studies. http://gwadi.org/ sites/gwadi.org/files/WestUS.pdf (Last access 18/09/ 2017).

IGME-OAPN. (2013). Guía geológica del Parque Nacional de Ordesa y Monte Perdido. Guías Geológicas de Parques Nacionales. Instituto Geológico y Minero de EspañaOrganismo Autónomo de Parques Nacionales, Madrid. Editorial Everest: 1-214.

Iribar, V., Antigüedad, I. (1996). Definición de zonas de recarga de manantiales kársticos mediante técnicas isotópicas ambientales. En: Simposio sobre Recursos Hidráulicos en Regiones Kársticas, Vitoria-Gasteiz. Gob. Vasco/AlH-GE: 271-280.

Jódar, J., Lambán. L.J., Medina, A., Custodio, E. (2014). Exact analytical solution of the convolution integral for classical hydrogeological lumped-parameter models and typical input tracer functions in natural gradient systems. Journal of Hydrology, 519: 3275-3289.

Jódar, J., Custodio, E., Liotta, M., Lambán, L.J., Herrera, C., Martos-Rosillo, S., Sapriza, G., Rigo, T. (2016a). Correlation of the seasonal isotopic amplitude of precipitation with annual evaporation and altitude in alpine regions. Science of the Total Environment, 550: 27-37.

Jódar, J., Custodio, E., Lambán, L.J., Martos-Rosillo, S., Herrera-Lameli, C., Sapriza-Azuri, G. (2016b). Vertical variation in the amplitude of the seasonal isotopic content of rainfall as a tool to jointly estimate the groundwater recharge zone and transit times in the Ordesa and Monte Perdido National Park aquifer system, north-eastern Spain. Science of the Total Environment, 573, 505-517.

Kabeya, N., Katsuyama, M., Kawasaki, M., Ohte, N., Sugimoto, A. (2007). Estimation of mean residence times of subsurface waters using seasonal variation in deuterium excess in a small headwater catchment in Japan. Hydrological Processes, 21(3): 308-322.

Kiraly, L. (1997) Modelling karst aquifers by the combined discrete channel and continuum approach. 6th Conference on Limestone Hydrology and Fissured Aquifers: Modelling Karst Aquifers. Université de Franche-Comté, Sciences et Techniques de I'Environnement, La Chaux-de-Fonds: 1-26.

Lambán, L.J., Jódar, J., Custodio, E. (2014). Caracterización hidrogeoquímica del agua subterránea en el Parque Nacional de Ordesa y Monte Perdido (Pirineo Central, España). En: J. Gómez-Hernández y J. Rodrigo-llarri (eds.), CIAS 2014. II Congreso Ibérico de las Aguas Subterráneas. Valencia. 2014. Ed. Universitat Politècnica de València: 487-497.

Lambán, L.J., Jódar, J., Custodio, E., Soler, A., Sapriza, G., Soto, R. (2015). Isotopic and hydrogeochemical characterization of high-altitude karst aquifers in complex geological settings. The Ordesa and Monte Perdido National Park (Northern Spain) case study. Science of the Total Environment, 506: 466-479.

Leibundgut, C., Maloszewski, P., Külls, C. (2011). Tracers in hydrology. John Wiley \& Sons.

Małoszewski, P., Zuber, A. (1982). Determining the turnover time of groundwater systems with the aid of environmental tracers, I. Models and their applicability. Journal of Hydrology 57, 207-231.

Martínez de Pisón, E (2002) Informe sobre la ampliación del Parque Nacional de Ordesa y Monte Perdido. Organismo Autónomo de Parques Nacioneles (MMA), Madrid, 117-141.

Moreno, A., Bartolomé, M., Pérez, C., Sancho, C., Cacho, I., Stoll, H., Delgado-Huertas, A., Edwards, R.L., Cheng,H. (2015). Tracking the origin of $\delta 180$ variability in speleothems: examples from the Ordesa and Monte Perdido National Park (NE Iberia). In : JP Galve, JM Azañón, JV. Pérez Peña, \& P Ruano (eds.), Una Visión Global del Cuaternario. El Hombre como Condicionante de Procesos Geológicos. Reunión Nacional de Cuaternario, Granada (España): 82-85.

Motyka, J. (1998). A conceptual model of hydraulic networks in carbonate rocks, illustrated by exemples from Poland. Hydrogeology Journal, 6: 469-482.

Muñoz, J.A., Martinez, A., Vergas, J. (1986). Thrust sequences in the Spanish eastern Pyrenees. Journal of Structural Geology, 8, p. 399-405.

Muñoz, J.A. (1992). Evolution of a continental collision belt: ECORS-Pyrenees cristal balanced cross-section. In: Thrust Tectonics (Ed. by K.R. McClay), Chapman\&Hall, 235-246.

Parish, M. (1984). A structural interpretation of a section of the Gavarnie nappe and its implications for Pyrenean geology. Journal of Structural Geology, 6, p. 247-255.

Peel, M.C., Finlayson, B.L., McMahon, T.A. (2007). Updated world map of the Köppen-Geiger climate classification. Hydrology of Earth System Science: 11: 1633-44.

Poage, M.A., Chamberlain, C.P. (2001). Empirical relationships between elevation and the stable isotope composition of precipitation and surface waters: considera- 
tions for studies of paleoelevation change. American Jounal of Science, 301(1): 1-15.

Polo, E. (2015). Uso y manejo de nuevas tecnologías y herramientas SIG a partir de datos hidrogeológicos en el Parque Nacional de Ordesa y Monte Perdido. Unpublished Master's Thesis. Department of Geography and Regional Planning. University of Zaragoza.

Rada-Martínez, B., Marquina-Murlanch, L., Viñuales-Cobos, E. (2011). Guía de visita del Parque Nacional de Ordesa y Monte Perdido. Ed. Organismo Autónomo Parques Nacionales, Madrid: 1-207.

Ríos-Aragüés, L.M. (2003). Introducción al mapa geológico del Parque Nacional de Ordesa y Monte Perdido. Sociedad Española de Espeleología y Ciencias del Karst (SEDECK), Boletín 5: 84-99.

Samper, J.; Huguet, LL. Ares, J., García-Vera, M.A. (2005). User's guide VisualBALAN v.2.0: código interactivo para la realización de balances hidrológicos y la estimación de la recarga. Escuela Técnica Superior de Ingenieros de Caminos, Canales y Puertos, A Coruña: 1-150.

Sapriza-Azuri G., Jódar J., Carrera J., Gupta HV. (2013). Stochastic simulation of nonstationary rainfall fields, accounting for seasonality and atmospheric circulation pattern evolution. Mathematical Geosciences, 45(5): $621-645$.

Sapriza Azuri, G., Jódar, J., Navarro, V., Slooten, L.J., Carrera, J., Gupta, H.V. (2015). Impacts of rainfall spatial variability on hydrogeological response. Water Resources Research, 51(2): 1300-1314.

Schaefer, R.W., and Usdowski, E. (1992). Application of stable carbon and sulfur isotope models to the development of groundwater in a limestone-dolomite-anhydrite-gypsum area. Progress in Hydrogeochemistry (G. Matthess, G.F. Frimmel, P. Hirsch, H.D., Schulz, H.-E., Usdowski, E., Eds), Springer, Berlin (1992) 157-163.

Seguret, M. (1972): Étude tectonique des nappes et séries décollées de la partie centrale du versant sud des Pyrénées. Pub. USTELA, Ser. Geol. Struct. n.2, Montpellier.

Seibert, J. (1997). Estimation of parameter uncertainty in the HBV model. Nordic Hydrology, 28(4), 247-262.).

Seibert, J. (2005). HBV light version 2, User's Manual. Uppsala University, Institute of Earth Sciences, Department of Hydrology, Uppsala, Sweden.

StichlerW, Schotterer U, Fröhlich K, Ginot P, Kull C, Gäggeler H, Pouyaud, B. (2001). Influence of sublimation on stable isotope records recovered from high-altitude glaciers in the tropical Andes. Journal of Geophysical Research, 106(D19): 22613-22620.

Zuber, A. (1986). Mathematical models for the interpretation of environmental radioisotopes in groundwater systems. In: P Fritz \& JC Fontes (eds.), Handbook of Environmental Isotope Geochemistry, vol. 2, Terrestrial Environment B, Elsevier: Amsterdam: 1-59.

Recibido: julio 2018

Revisado: septiembre 2018

Aceptado: febrero 2019

Publicado: diciembre 2019 